\title{
Liver Cancer Study Group of Japan Clinical Practice Guidelines for Intrahepatic Cholangiocarcinoma
}

\author{
Shoji Kubo ${ }^{a}$ Hiroji Shinkawa $^{a}$ Yoshinari Asaoka $^{\mathrm{b}}$ Tatsuya loka $^{\mathrm{c}}$

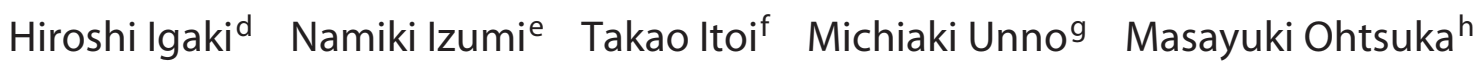 \\ Takuji Okusakai Masumi Kadoyaj Masatoshi Kudok Takashi Kumadal \\ Norihiro Kokudo $^{m}$ Michiie Sakamoto ${ }^{\mathrm{n}}$ Yoshihiro Sakamoto $^{\circ}$ \\ Hideyuki Sakurai $^{p}$ Tadatoshi Takayama ${ }^{q}$ Osamu Nakashimar \\ Yasushi Nagata $^{\mathrm{s}}$ Etsuro Hatano ${ }^{\mathrm{t}}$ Kenichi Harada ${ }^{\mathrm{u}}$ Takamichi Murakami $^{\mathrm{v}}$ \\ Masakazu Yamamotow

\begin{abstract}
aDepartment of Hepato-Biliary-Pancreatic Surgery, Osaka City University Graduate School of Medicine, Osaka, Japan; ${ }^{b}$ Department of Medicine, Teikyo University School of Medicine, Tokyo, Japan; 'Department of Oncology Center, Yamaguchi University Hospital, Yamaguchi, Japan; ${ }^{d}$ Department of Radiation Oncology, National Cancer Center Hospital, Tokyo, Japan; ' Department of Gastroenterology and Hepatology, Musashino Red Cross Hospital, Tokyo, Japan; fDepartment of Gastroenterology and Hepatology, Tokyo Medical University Hospital, Tokyo, Japan; ${ }^{9}$ Department of Surgery, Tohoku University School of Medicine, Miyagi, Japan; 'hepartment of General Surgery, Graduate School of Medicine, Chiba University, Chiba, Japan; 'Department of Hepatobiliary and Pancreatic Oncology, National Cancer Center Hospital, Tokyo, Japan; 'Department of Radiology, Shinshu University School of Medicine, Matsumoto, Japan; kDepartment of Gastroenterology and Hepatology, Kindai University Faculty of Medicine, Osaka, Japan; 'Department of Gastroenterology and Hepatology, Ogaki Municipal Hospital, Gifu, Japan; ${ }^{m}$ Department of Surgery, National Center for Global Health and Medicine, Tokyo, Japan; 'Department of Pathology, Keio University School of Medicine, Tokyo, Japan; 'Department of Hepato-Biliary-Pancreatic Surgery, Kyorin University Hospital, Tokyo, Japan; PDepartment of Radiation Oncology, Faculty of Medicine, University of Tsukuba, Ibaraki, Japan; 'Department of Digestive Surgery, Nihon University School of Medicine, Tokyo, Japan;

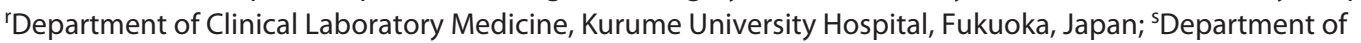
Radiation Oncology, Graduate School of Biomedical Sciences, Hiroshima University, Hiroshima, Japan; ${ }^{\text {DDepartment }}$ of Gastroenterological Surgery, Hyogo College of Medicine, Hyogo, Japan; "Department of Human Pathology, Kanazawa University Graduate School of Medicine, Ishikawa, Japan; vDepartment of Radiology, Kobe University Graduate School of Medicine, Hyogo, Japan; 'Department of Surgery, Institute of Gastroenterology, Tokyo Women's Medical University, Tokyo, Japan
\end{abstract}

\section{Keywords}

Intrahepatic cholangiocarcinoma - Guidelines - Etiology · Pathology · Diagnosis · Treatment

\begin{abstract}
This paper presents the first version of clinical practice guidelines for intrahepatic cholangiocarcinoma (ICC) established by the Liver Cancer Study Group of Japan. These guidelines consist of 1 treatment algorithm, 5 background statements, 16
\end{abstract}


clinical questions, and 1 clinical topic, including etiology, staging, pathology, diagnosis, and treatments. Globally, a high incidence of ICC has been reported in East and Southeast Asian countries, and the incidence has been gradually increasing in Japan and also in Western countries. Reported risk factors for ICC include cirrhosis, hepatitis B/C, alcohol consumption, diabetes, obesity, smoking, nonalcoholic steatohepatitis, and liver fluke infestation, as well as biliary diseases, such as primary sclerosing cholangitis, hepatolithiasis, congenital cholangiectasis, and Caroli disease. Chemical risk factors include thorium-232, 1,2-dichloropropane, and dichloromethane. CA19-9 and CEA are recommended as tumor markers for early detection and diagnostic of ICC. Abdominal ultrasonography, CT, and MRI are effective imaging modalities for diagnosing ICC. If bile duct invasion is suspected, imaging modalities for examining the bile ducts may be useful. In unresectable cases, tumor biopsy should be considered when deemed necessary for the differential diagnosis and drug therapy selection. The mainstay of treatment for patients with Child-Pugh class A or $B$ liver function is surgical resection and drug therapy. If the patient has no regional lymph node metastasis (LNM) and has a single tumor, resection is the treatment of choice. If both regional LNM and multiple tumors are present, drug therapy is the first treatment of choice. If the patient has either regional LNM or multiple tumors, resection or drug therapy is selected, depending on the extent of metastasis or the number of tumors. If distant metastasis is present, drug therapy is the treatment of choice. Percutaneous ablation therapy may be considered for patients who are ineligible for surgical resection or drug therapy due to decreased hepatic functional reserve or comorbidities. For unresectable ICC without extrahepatic metastasis, stereotactic radiotherapy (tumor size $\leq 5 \mathrm{~cm}$ ) or particle radiotherapy (no size restriction) may be considered. ICC is generally not indicated for liver transplantation, and palliative care is recommended for patients with Child-Pugh class $C$ liver function.

(c) 2022 The Author(s)

Published by S. Karger AG, Basel

\section{Introduction}

The Liver Cancer Study Group of Japan has established this first version of clinical practice guidelines for intrahepatic cholangiocarcinoma (ICC) according to the methodology of evidence-based medicine, including the Grading of Recommendations Assessment, Development, and Evaluation system. Scientific papers published as of the end of 2019 in the PubMed database were reviewed, along with some important papers written in Japanese. The contents of the guidelines were determined in

Clinical Practice Guidelines for

Intrahepatic Cholangiocarcinoma accordance with the national health insurance system in Japan. The guidelines consist of 1 treatment algorithm, 5 background statements (BSs), 16 clinical questions (CQs), and 1 clinical topic, including etiology, staging, pathology, diagnosis, and treatments.

\section{Treatment Algorithm for ICC}

The treatment algorithm for ICC was developed based on four factors: hepatic functional reserve, distant metastasis, regional lymph node (LN) metastasis (LNM), and number of tumors (single or multiple) (Fig. 1). The risk factors for ICC include cirrhosis and hepatitis B/C, meaning that patients with ICC often have liver damage. Therefore, it is necessary to assess hepatic functional reserve. Currently, the mainstay of treatment for patients classified with Child-Pugh class A or B liver function is surgical resection and drug therapy. In these patients without distant metastasis (including the extra-regional LNM), the treatment is selected according to the presence or absence of regional LNM and the number of tumors (single or multiple). If the patient has no regional LNM and has a single tumor, resection is the treatment of choice. If both regional LNM and multiple tumors are present, drug therapy is the first treatment of choice. If the patient has either regional LNM or multiple tumors, resection or drug therapy is selected depending on the extent of metastasis or the number of tumors. If distant metastasis is present, drug therapy is the treatment of choice.

Percutaneous ablation therapy may be considered for patients who are ineligible for surgical resection or drug therapy, owing to decreased hepatic functional reserve or comorbidities. For unresectable ICC without extrahepatic metastasis, stereotactic radiotherapy (tumor size $\leq 5$ $\mathrm{cm}$ ) or particle radiotherapy (no size restriction) may be considered. ICC is generally not indicated for liver transplantation, and palliative care is recommended for patients with Child-Pugh class $\mathrm{C}$ liver function.

\section{Background Statements}

BS 1: Change in the Incidence of ICC Worldwide and Its Regional Characteristics

Comments

Globally, a high incidence of ICC has been reported in East and Southeast Asian countries. In recent years, the incidence has been gradually increasing in Japan and also in Western countries. 
Fig. 1. Treatment algorithm for ICC (massforming and mass-forming predominant types).

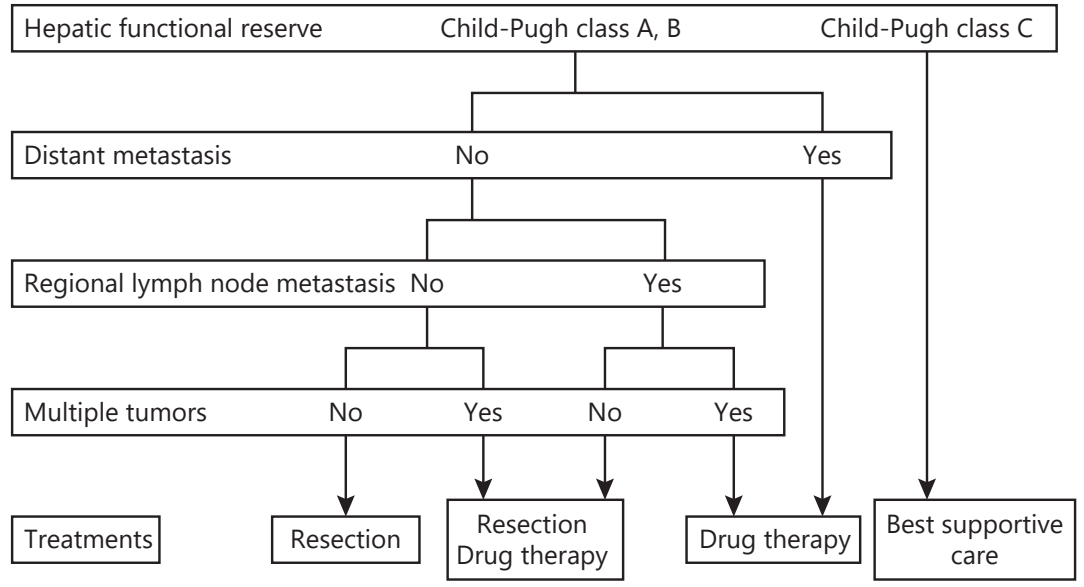

Local ablation therapy may be considered for patients who are ineligible for surgical resection or drug therapy due to decreased hepatic functional reserve or comorbidities. For unresectable intrahepatic cholangiocarcinoma without extrahepatic metastasis, stereotactic radiotherapy $(\leq 5 \mathrm{~cm})$ or particle radiotherapy (no size restriction) may be considered.

Table 1. Trends in the incidence of ICC

\begin{tabular}{ll}
\hline Direction of trend & Countries \\
\hline Upward & USA, ${ }^{5}$ Italy, ${ }^{12}$ Australia, ${ }^{12}$ Austria, ${ }^{7}$ Netherlands, ${ }^{6}$ Canada, ${ }^{11}$ Thailand,${ }^{4}$ Taiwan, $^{2}$ Japan, ${ }^{8}$ France \\
Downward & South Korea, ${ }^{3}$ Denmark
\end{tabular}

\section{Description}

The overall incidence of cholangiocarcinoma is high in East and Southeast Asia, with the highest incidence reported in Northeast Thailand, where the incidence in men is 85 cases per 100,000 [1]. Although age-adjusted incidence rates vary depending on the reference population and thus cannot be simply compared among countries, the incidence of ICC is increasing worldwide, as seen from the trends in various countries (Table 1).

The age-adjusted incidence rate of ICC in Taiwan was 2.19 cases per 100,000 person-years in 2008 , showing an approximately 3 -fold increase over the previous decade [2]. In South Korea, the corresponding rate was 7.8 cases per 100,000 person-years in 2015 and had shown an upward trend until 2010 and then a downward trend until 2015 [3]. The incidence has also been rising in Western countries in recent years. In the USA, the age-adjusted incidence rate was 1.18 cases per 100,000 person-years in 2012 , showing an increase of $4.36 \%$ since 1973 [4]. In the Netherlands, the corresponding rate was 0.35 cases per
100,000 person-years in 2009 , an increase of $9.4 \%$ since 2000 [5]. In 2000, the 3rd edition of the International Classification of Diseases for Oncology was published, and many Klatskin tumors (hilar cholangiocarcinoma), which had been classified as ICC in the 1st and 2nd editions, were excluded from the ICC category, owing to registration of accurate localization. Even taking this into account, the incidence of ICC is on the rise worldwide $[1,6$, 7].

The average age-adjusted incidence rate of ICC in Japan showed increases of $9.1 \%$ in males from 1985 to 2007 and $12.9 \%$ in females from 1985 to 1998 [6]. Similarly, the rate showed respective increases of $1.8 \%$ for males and $1.2 \%$ for females from 1996 to 2011 [8]. A report from the Japanese Ministry of Health, Labour and Welfare has noted that the rate for ICC has been increasing in recent years [9]. The Report of the 20th National Follow-up Survey on Primary Liver Cancer (2008-2009) by the Liver Cancer Study Group of Japan (LCSGJ) indicated that ICC accounts for $4.77 \%$ of all primary liver cancers [10]. 
BS 2: Risk Factors for the Development of ICC

Comments

Reported risk factors for ICC include cirrhosis, hepatitis $\mathrm{B} / \mathrm{C}$, alcohol consumption, diabetes, obesity, smoking, nonalcoholic steatohepatitis, and liver fluke infestation, as well as biliary diseases, such as primary sclerosing cholangitis (PSC), hepatolithiasis, congenital cholangiectasis (choledochal cyst), Caroli disease, and inflammatory bowel disease. Chemical risk factors include thorium-232, 1,2-dichloropropane, and dichloromethane.

\section{Description}

Epidemiological information on risk factors for ICC is often derived from case-control studies, and the possibility of selection bias for the regions and populations must be considered. It has also been suggested that the revision of the International Classification of Diseases may have affected the results of epidemiological studies on risk factors. As described in the Cancer Incidence in Five Continents published by the International Agency for Research on Cancer, the incidence of ICC is higher in men than in women [11]. It should however be noted that the malefemale ratio varies among countries and regions. The incidence is higher among Chinese and Filipinos living in Hawaii than among Whites living in Hawaii, suggesting that Asians have a higher incidence of ICC than do other races $[11,12]$. These racial differences may be related to lifestyle, diet, and region-specific diseases.

Conventional risk factors for ICC include diseases associated with persistent cholangitis and chemical substances, although recent studies have identified liver damage due to hepatitis or other causes as an additional risk factor for ICC [13]. The association between viral hepatitis and ICC has been extensively documented. A meta-analysis of 26 studies examining the incidence of chronic hepatitis B and ICC reported an odds ratio (OR) of 3.1 [14]. In Japan, chronic hepatitis $C$ has been recognized as a risk factor for ICC since reports by Kobayashi et al. [15] and Yamamoto et al. [16] were published in the early 2000s. In fact, a meta-analysis of 16 studies examining the incidence of chronic hepatitis C and ICC reported an OR of 3.3 [17]. The Report of the 20th National Follow-up Survey showed that among all patients with ICC, $8.2 \%$ were positive for HBs antigen, and $14.9 \%$ were positive for hepatitis C virus antibody [8]. Cirrhosis is also a risk factor for ICC, regardless of its etiology, with an OR of 22.9 according to a meta-analysis of 11 studies [18]. Commonly reported risk factors include alcohol consumption (OR 2.8) and metabolic factors such as diabetes
(OR 1.8) and obesity (OR 1.5) [18]. The association between smoking and ICC was demonstrated in a prospective cohort study of $1,518,741$ patients conducted in the USA, with a hazard ratio (HR) of 1.4 in smokers [19]. Recently, nonalcoholic steatohepatitis has attracted attention as a risk factor for cholangiocarcinoma, including ICC [20].

Meta-analyses of liver flukes (Clonorchis sinensis and Opisthorchis viverrini) as a risk factor for cholangiocarcinoma showed ORs of 4.7 for $C$. sinensis and 6.4 for $O$. viverrini $[21,22]$. The association between hepatolithiasis and ICC has been reported in case-control studies from Taiwan [23] and South Korea [24] with ORs ranging from 4.8 to 50 . The same association has also been reported in Japan [25], with the reported rate of coexistence of hepatolithiasis and ICC ranging from $1.3 \%$ to $11 \%$ [26]. PSC is prevalent in Western countries and is an important risk factor for ICC [27]. Inflammatory bowel disease has been also reported to be associated with the occurrence of ICC [28].

An association has also been documented between ICC and biliary cystic diseases, including congenital cholangiectasis (choledochal cyst), pancreaticobiliary maljunction, and Caroli disease. A case-control study in the USA showed ORs of 15.6 for choledochal cyst and 38.1 for Caroli disease [29]. Thorium-232 is a known risk factor for ICC, with an OR of 23.3-316 [30].

Recently, a number of cases of cholangiocarcinoma, including ICC, were discovered among employees of a printing company, revealing the causal relationship between the occurrence of cholangiocarcinoma and highdose long-term exposure to 1,2-dichloropropane and/or dichloromethane; they also had a high incidence of genetic mutations [31,32]. The standardized incidence of cholangiocarcinoma due to exposure to 1,2-dichloropropane and both 1,2-dichloropropane and dichloromethane were 1,002.8 and 1,319.9 per 100,000 person-years, respectively [33].

\section{BS 3: Differences in Staging Criteria for ICC between Japan and Western Countries \\ Comments}

The revised staging criteria for ICC in the General Rules for the Clinical and Pathological Study of Primary Liver Cancer (6th edition) were based on the results of multivariate analysis of prognostic factors in surgically resected cases in Japan. The staging criteria are different from those in the UICC, 8th edition in some respects. 
Table 2. Staging criteria for ICC in the general rules for the clinical and pathological study of primary liver cancer (revised 6th edition)

\begin{tabular}{|c|c|c|c|c|}
\hline Stage/factors & $\mathrm{T}$ & $\mathrm{N}$ & M & \\
\hline Stage I & $\mathrm{T} 1$ & No & Mo & \\
\hline Stage II & $\mathrm{T} 2$ & N1 & M0 & \\
\hline Stage III & T3 & N2 & Mo & \\
\hline Stage IVA & $\begin{array}{l}\text { T4 } \\
\text { Other than T4 }\end{array}$ & $\begin{array}{l}\text { N3 } \\
\text { N1 }\end{array}$ & $\begin{array}{l}\text { M0 } \\
\text { M0 }\end{array}$ & \\
\hline Stage IVB & $\begin{array}{l}\text { T4 } \\
\text { Any T }\end{array}$ & $\begin{array}{l}\text { N1 } \\
\text { N0, N1 }\end{array}$ & $\begin{array}{l}\text { M0 } \\
\text { M1 }\end{array}$ & \\
\hline T-stages of ICC & $\mathrm{T} 1$ & $\mathrm{~T} 2$ & T3 & $\mathrm{T} 4$ \\
\hline $\begin{array}{l}\text { (1) Number, solitary } \\
\text { (2) Size, }<2 \mathrm{~cm} \\
\text { (3) No vascular invasion or major biliary invasion ( } \mathrm{Vp} 0, \mathrm{VaO}, \mathrm{BO}-2)\end{array}$ & $\begin{array}{l}\text { Match all } \\
\text { three factors }\end{array}$ & $\begin{array}{l}\text { Match } \\
2 \text { factors }\end{array}$ & $\begin{array}{l}\text { Match } \\
1 \text { factor }\end{array}$ & $\begin{array}{l}\text { No } \\
\text { matching }\end{array}$ \\
\hline
\end{tabular}

\section{Description}

Currently, the most commonly used staging criteria for ICC are based on the General Rules for the Clinical and Pathological Study of Primary Liver Cancer (Revised 6th Edition) issued by the LCSGJ [34] and the UICC's TNM Classification of Malignant Tumours (8th Edition) [35]. For revision in the 6th edition, 419 surgically resected cases of mass-forming ICCs were collected through the National Follow-up Survey (14th-18th) by the LCSGJ [36]. Multivariate analysis of histopathological factors was performed to reveal significant factors in survival to use as staging criteria. Briefly, T-stage was defined by tumor size, number of tumors, and the presence of vascular invasion of the hepatic artery or portal vein or major biliary invasion. Patients with nodal metastasis (stage IV in the 5 th edition) were subclassified into stage IVA with T1-T3 and stage IVB with T4 or distant metastasis (Table 2) [37]. When the new staging criteria were used to generate the survival curves of the 419 patients, the differences between the curves of adjacent stages became more significant than those in 5th edition (Fig. 2).

The cutoff value of tumor size for determining the Tstage is $2 \mathrm{~cm}$, and there was a clear difference in prognosis between the $\leq 2$ - $\mathrm{cm}$ cohort and the $>2$ - $\mathrm{cm}$ cohort. In contrast, no significant difference was observed with a cutoff value of $5 \mathrm{~cm}$. Of the 419 patients evaluated, 27 had small ICCs $\leq 2 \mathrm{~cm}$ in size, and 15 of these patients without nodal metastasis or portal vein invasion had a $100 \% 5$-year survival rate. Thus, the $2-\mathrm{cm}$ tumor size cutoff is used as a criterion for the T-stage, as in the staging criteria for hepatocellular carcinoma (HCC). This stands in contrast to the re-inclusion of the 5 - $\mathrm{cm}$ cutoff as a criterion for the T-stage in the UICC (8th edition). To clarify the significance of the cutoff value of $2 \mathrm{~cm}$, further validation is necessary. In addition, the presence of major biliary invasion is used as a criterion for the T-stage. Pathological biliary invasion can be classified from b0 to b4 (Table 3 ).

The current revision is based on the observation that in 267 patients without nodal metastasis or distant metastasis, univariate and multivariate analyses revealed that b3 or b4 biliary invasion was an independent and significant prognostic factor. This indicates that involvement of first-order bile duct branches or the common bile duct (major biliary invasion) is associated with poor prognosis of ICC. Clinically, this is consistent with the observation that ICC involving the hepatic hilum has poorer prognosis than peripheral ICC. Moreover, mass-forming plus periductal-infiltrating type ICC has poorer prognosis than mass-forming type ICC [38]. However, there have been few reports showing the poor prognostic impact of major biliary invasion, and thus, the accumulation of further evidence is necessary.

In the UICC 8th edition, the T-stage is defined by the number of tumors (single vs. multiple), tumor size $(\leq 5 \mathrm{~cm}$ vs. $>5 \mathrm{~cm}$ ), and the presence of vascular/serosal invasion or invasion of other organs. Periductal invasion, which constituted the criteria for T4 staging in the 7th edition, was removed in the 8th edition (Table 4) [35]. The pres- 


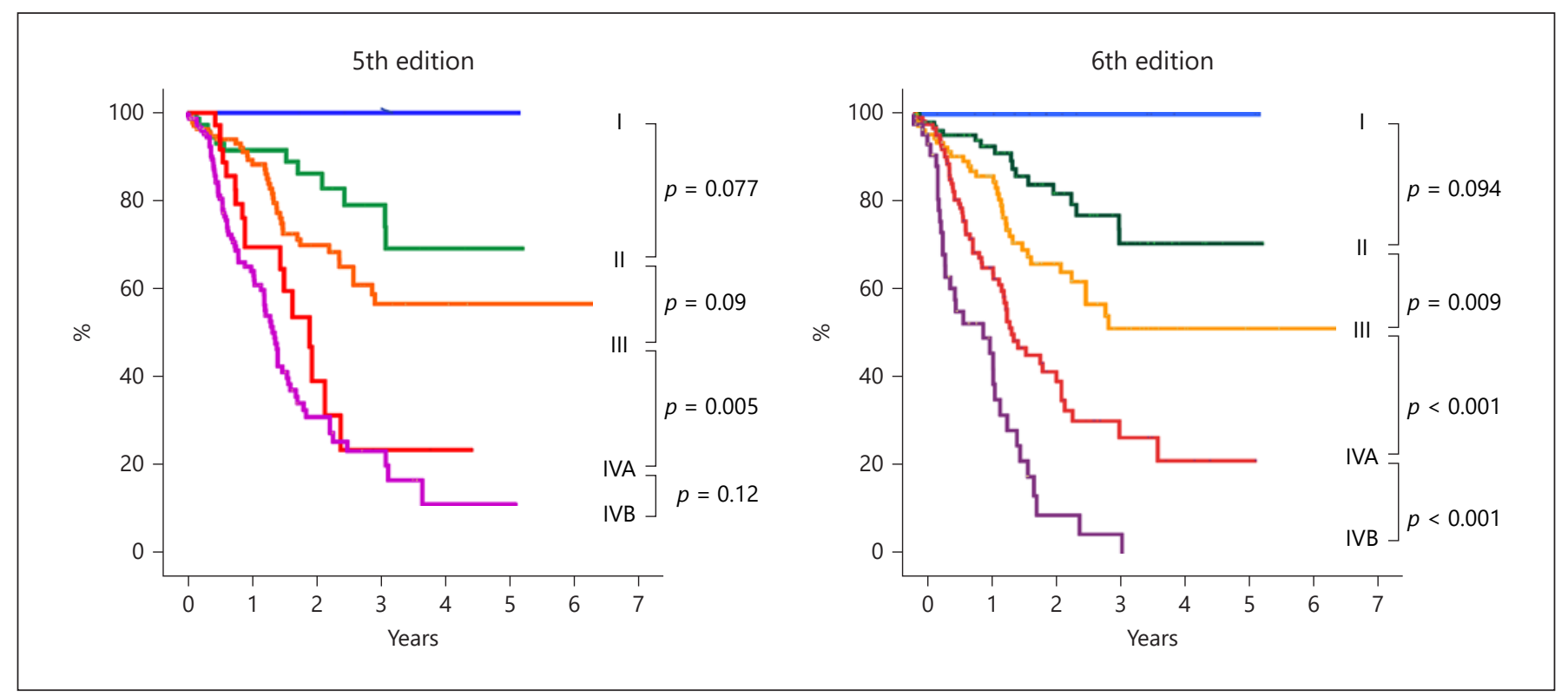

Fig. 2. Survival curves according to staging criteria in the 5 th and 6 th editions of the general rules for the Clinical and Pathological Study of Primary Liver Cancer.

Table 3. Bile duct invasion

b0 Invasion of the bile ducts undetected

b1 Invasion of (or tumor thrombus in) the third-order or more peripheral branches of the bile duct but not of second-order branches

b2 Invasion of (or tumor thrombus in) the second-order branches of the bile duct detected

b3 Invasion of (or tumor thrombus in) the first-order branches of the bile duct detected

b4 Invasion of (or tumor thrombus in) the common hepatic duct detected

ence of serosal invasion, which is included in the 8th edition, was not an independent prognostic factor in an analysis of surgically resected cases in Japan [39]. Furthermore, although the definition of T2 includes both a solitary tumor with vascular invasion and multiple tumors, the latter was clearly associated with a poorer prognosis than the former in surgically resected cases. Thus, it is difficult to simply extrapolate the criteria in the UICC 8 th edition to clinical practice in Japan. The criteria are not intended for Japanese patients, and the treatment of ICC in Japan is different from that in the USA and Europe in some respects.

BS 4: Precancerous and Early-Stage Cancerous Lesions of ICC

Comments

Precancerous and early-stage cancerous lesions include biliary intraepithelial neoplasia (BilIN), intraductal papillary neoplasm of bile duct (IPNB), and mucinous cystic neoplasm (MCN; mucinous cystadenoma/cystadenocarcinoma). Microscopically identified lesions known as biliary intraepithelial dysplasia or biliary intraepithelial dysplastic lesions, as well as biliary papilloma(tosis), mucin-producing biliary tumors, and biliary cystic adenoma/adenocarcinoma communicating with the dilated bile ducts, have been organized into BilIN and IPNB according to the 2010 and 2019 WHO Classification of Tumours of the Digestive System [40, 41].

\section{Description}

BilIN [40-43]: A microscopically identified neoplastic lesion of the biliary epithelium, with a flat or micropapillary morphology, is classified according to the degree of cytoarchitectural atypia as BilIN-1, BilIN-2, or BillN-3, which correspond to mild, moderate, and severe dysplasia (including carcinoma in situ), respectively. With in- 
Table 4. Staging criteria for ICC in the UICC, 8th edition

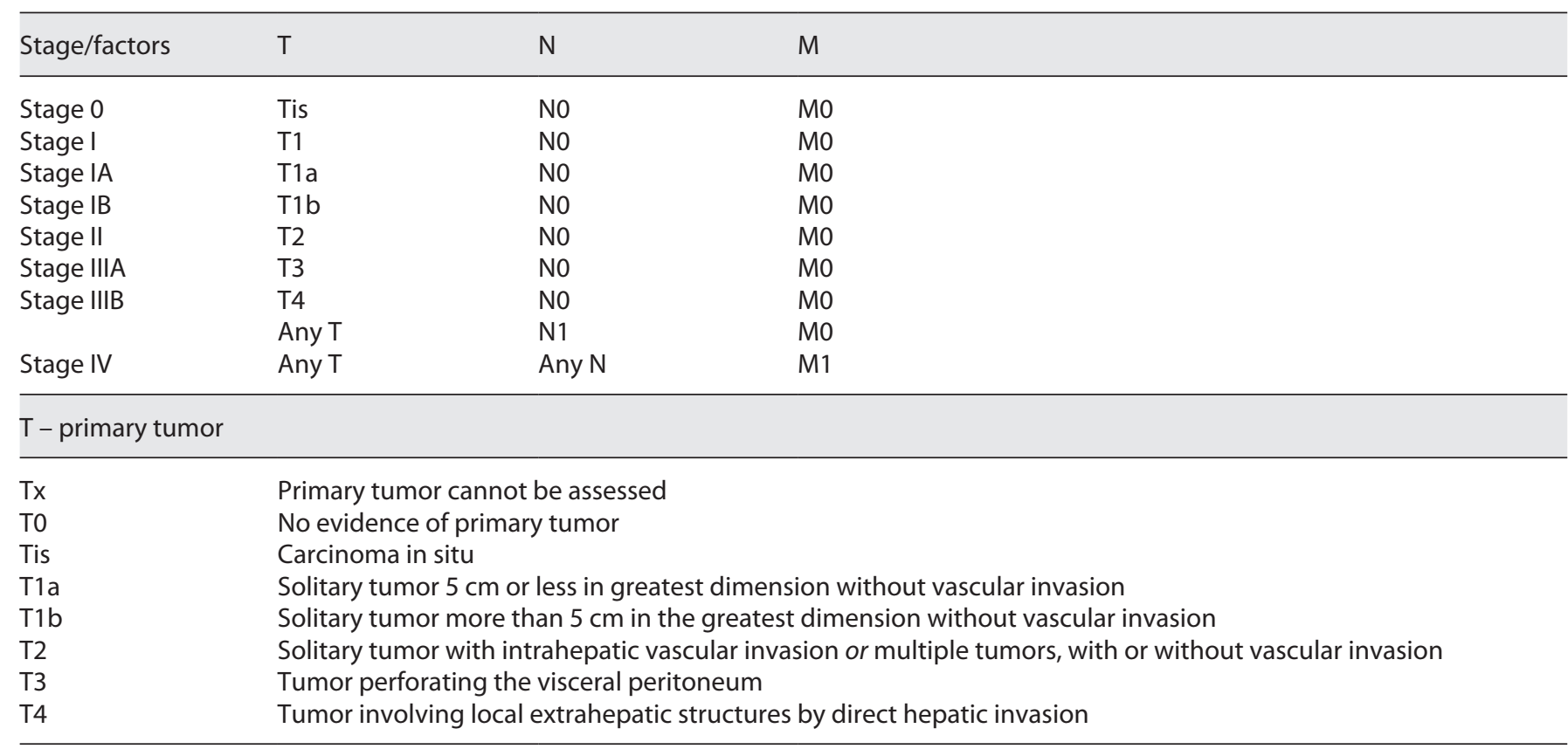

creasing severity, there is increasing expression of cell cycle proteins and an increasing frequency of gene mutations. The 2019 WHO Classification [41] recommends that BilIN-1 and BilIN-2 be classified as low grade and BilIN-3 as high grade. These lesions are commonly observed in patients with chronic cholangitis with hepatolithiasis and in the biliary epithelium in ICC.

IPNB [40-42, 44, 45]: A macroscopically identified papillary neoplastic lesion, with dilatation of the bile ducts around the tumor, in some cases showing cystic or mass-like dilatation. The 2019 WHO Classification classifies IPNB into type 1 and type 2 based on the histological features reported by a Japan-Korea joint expert panel [44]. In most cases, IPNB typically shows communication with the bile ducts. IPNB is often accompanied by mucus hypersecretion. Microscopically, it appears as a papillary or villous proliferation of epithelium with a fine fibrovascular core and tubular or glandular components. Neoplastic epithelium can be classified into the pancreatic/ biliary, intestinal, oncocytic, or gastric types. They can also be classified according to the degree of cytoarchitectural atypia: mild, moderate (borderline lesions), severe (including carcinoma in situ), or sometimes mixed. The 2019 WHO Classification [41] classifies mild to moderate atypia (borderline lesions) as IPNB with low-grade intraepithelial neoplasia and classifies severe atypia (in- cluding carcinoma in situ) as IPNB with high-grade intraepithelial neoplasia. There are many cases in which neoplastic epithelial cells in the bile ducts show superficial spread along the mucosal surface of the bile duct in contact with the papillary neoplasm. Neoplastic epithelial cells can also invade the bile duct wall as IPNB with associated invasive carcinoma, with occasional colloid carcinoma or tubular adenocarcinoma observed in the parenchymal invasion. To differentiate IPNB from MCN of the liver, it is important to confirm the absence of ovarian-like hypercellular stroma in the wall and the presence of communication with the bile duct.

MCN [40-42] (mucinous cystadenoma/cystadenocarcinoma): A unilocular or multilocular cystic neoplasm with a clear fibrous capsule containing clear, hemorrhagic, or mucous fluid. MCN occurs predominantly in women. It is generally considered to have no communication with the bile ducts. Microscopically, the inner surface of the cyst is lined with papillary or flat neoplastic cuboidal to columnar epithelium. The cytoplasm appears pale and is often positive for mucus staining. Characteristic findings include an ovarian-like hypercellular stroma in the subepithelial cyst wall and progesterone and estrogen receptor expression in the stromal cells. Depending on the degree of atypia, these lesions can be classified into mild to moderate atypia or severe atypia (including carcinoma 
in situ). Some MCNs have invasive carcinoma with a papillary or solid lesion. MCNs with mild to moderate atypia are referred to as mucinous cystadenoma, and those with severe atypia or invasive carcinomas are referred to as mucinous cystadenocarcinoma. The 2019 WHO Classification [41] defines mild to moderate atypia type as "MCN with low-grade intraepithelial neoplasia" and severe atypia, including carcinoma in situ, as "MCN with high-grade intraepithelial neoplasia." These lesions were previously referred to as biliary cystadenoma/cystadenocarcinoma with an ovarian-like hypercellular stroma in the bile duct wall.

\section{BS 5: Tumor-Like Conditions Arising from the}

\section{Intrahepatic Biliary Tract}

Comments

Intrahepatic pseudotumors [46-51] include PSC, IgG4-related sclerosing cholangitis, secondary sclerosing cholangitis, inflammatory pseudotumor, granuloma, liver abscess, solitary coagulation necrotic nodule, ductular reaction, bile duct adenoma (peribiliary gland hamartoma), Von Meyenburg complex, multicystic biliary hamartoma, peribiliary cyst, hepatolithiasis, ectopic tissue (ectopic pancreas, ectopic gastric mucosa).

\section{Description}

Intrahepatic pseudotumors, including PSC, IgG4-related sclerosing cholangitis, and secondary sclerosing cholangitis and inflammatory pseudotumors, should be differentiated from ICC. Small nodular lesions include ductular reaction and bile duct adenoma. Cystic lesions include von Meyenburg complex, multicystic biliary hamartoma, and peribiliary cyst. Other tumor-like lesions include hepatolithiasis and ectopic tissue.

\section{Clinical Questions}

\section{CQ 1: Is There an Effective Screening Method?}

\section{Recommendation}

No effective screening method has been established. However, patients with risk factors may need regular screening using liver function tests, tumor markers, and abdominal ultrasonography (US) (weak recommendation).

\section{Description}

Many patients with early-stage cholangiocarcinoma have no symptoms. However, as the disease advances, a variety of symptoms may appear. It is well known that general early warning signs of cholangiocarcinoma include jaundice (84\%-90\%), weight loss (35\%), abdominal pain $(30 \%)$, nausea/vomiting $(12 \%-25 \%)$, and fever $(10 \%)[52,53]$. Although there are only a few reports focusing on the initial symptoms of ICC, patients with ICC most frequently presented with jaundice (33.2\%), ascites (7.0\%), and encephalopathy ( $0.6 \%)$ at the time of diagnosis, according to the Report of the 20th National Followup Survey [8]. Early detection of cancer including ICC favors a greater chance of cure, longer survival, and higher quality of life for the patient. However, the appearance of symptoms may not contribute to early detection and diagnosis of ICC. Overall, we could not find a surveillance program for early detection and diagnosis of ICC [1].

As discussed in BS 2, many risk factors of ICC have been verified. For patients with risk factors such as hepatitis B [14], hepatitis C [17], cirrhosis [18], NASH [18], hepatolithiasis [25], and diabetes [18], it is prudent to add ICC to the list of differential diagnoses. Employees with a history of exposure to high concentrations of 1,2-dichloropropane for 2 years or more are issued a "health management handbook" by the Japanese Ministry of Health, Labour and Welfare, and they can receive a free health checkup once every 6 months at designated medical institutions, including measurement of serum $\gamma$-GTP and CA19-9 and imaging studies such as abdominal US [54].

\section{CQ 2: What Blood Tests Are Used to Detect ICC}

Recommendation

Persistent elevation of serum bilirubin and alkaline phosphatase can suggest the presence of malignant biliary stenosis (weak recommendation). CA19-9 and CEA are recommended as tumor markers for early detection and diagnosis of ICC (strong recommendation).

\section{Description}

Although bile duct stenosis/obstruction can lead to liver dysfunction and be accompanied by elevated bilirubin levels and hepatobiliary enzymes (e.g., ALP, $\gamma$-GTP, AST, ALT), high values of these factors may not contribute to detecting ICC. However, some studies have suggested that persistent elevation of serum bilirubin and ALP is associated with the presence of malignant biliary stenosis [55-59].

Biomarkers for cholangiocarcinoma such as CEA, CA19-9, DUPAN-2, SPan-1, NCC-ST-439, and TPA are covered by Japanese national health insurance. The positive rates of CA19-9 $(>37 \mathrm{U} / \mathrm{mL})$ and CEA $(>5 \mathrm{ng} / \mathrm{mL})$ were $54.1 \%$ and $33.6 \%$ in Japanese patients with ICC, re- 
spectively [8]. CEA and CA19-9 have high sensitivity and specificity for cholangiocarcinoma, respectively [60]. The combination of CA19-9 and CEA achieves better sensitivity and accuracy [60].

\section{CQ 3: What Imaging Modalities Are Effective for}

Diagnosing ICC?

Recommendation

Abdominal US, CT, and MRI are effective imaging modalities for diagnosing ICC (Strong recommendation).

\section{Description}

Mass-forming type ICC is a mass-like lesion with irregular margins and relatively indistinct borders, often accompanied by dilatation of the distal bile ducts [61]. On abdominal US, it is visualized as an irregular-shaped or lobulated mass with heterogeneous content [62]. Characteristic contrast-enhanced US findings are heterogeneous enhancement of the tumor margin in the arterial-dominant phase and a marked defect in the late phase [62]. The diagnostic performance of contrast-enhanced US for ICC is $60 \%-90 \%$ sensitivity and $65 \%-98 \%$ specificity $[63,64]$. However, tumors less than $3 \mathrm{~cm}$ in size may be completely enhanced and need to be differentiated from HCC [65].

Mass-forming type ICC is hypointense on noncontrast CT and shows a ring-like enhancement pattern in the early phase of contrast-enhanced CT. This enhancement pattern has diagnostic sensitivity and specificity of $60 \%$ and $65.5 \%$, respectively $[66,67]$. Note, however, that the enhancement pattern of ICCs less than $3 \mathrm{~cm}$ in size varies from ring-like to homogeneous [67]. A characteristic persistent enhancement pattern in the late phase is observed in $67 \%$ of cases and depends on the rich stromal component in the center of the tumor [66].

On MRI, the lesion is hypointense on T1-weighted images and hyperintense on T2-weighted images, and the findings of dynamic MRI using extracellular fluid contrast agents are similar to those of CT [68]. On contrastenhanced MRI with the hepatocellular contrast agent GdEOB-DTPA (EOB-MRI), the lesion shows findings similar to those of MRI using extracellular fluid contrast agents in the early phase, but in the transitional phase, it shows a pseudo-washout pattern due to the uptake of contrast agent by hepatocytes around the lesion [69]. Therefore, for effective differentiation between ICC and HCC, the washout pattern should be evaluated in the portal venous phase rather than in the transitional phase [66]. ICC cells do not express the OATP1b3 transporter, and the lesion therefore appears hypointense in the hepato- cellular phase of EOB-MRI. However, some tumors with significant fibrosis may show slight central enhancement $[69,70]$, and this concentric, slight central enhancement pattern is another characteristic of ICC [71-73]. On diffusion-weighted imaging, the lesion shows concentric hyperintensity in $29 \%-52 \%$ of cases $[71,73,74]$. For a liver tumor in a cirrhotic patient, it is necessary to distinguish between HCC and ICC, which is often difficult, owing to their similar findings [67]. The sensitivity and specificity of MRI for differentiating ICC from HCC are reported to be $68.8 \%-93.5 \%$ and $86.2 \%-97.7 \%$, respectively [71, 75, $76,77]$.

ICC frequently shows high FDG uptake, and the sensitivity and specificity of positron emission tomography (PET) for diagnosing mass-forming type ICCs measuring $\geq 1 \mathrm{~cm}$ in size are reported to be $100 \%$ and $85 \%-90 \%$, respectively [78-81]. However, even though FDG-PET is highly useful for differentiating between benign and malignant lesions, there is little evidence for its effectiveness in differentiating hepatic masses. Therefore, whether to perform FDG-PET in clinical practice should be determined by also considering its cost-effectiveness.

ICC lesions are often difficult to differentiate from mixed HCC-cholangiocarcinoma lesions. Findings such as dilated intrahepatic bile ducts, retraction of the hepatic capsule, and the appearance of the background liver may provide clues for differentiation $[76,82]$.

\section{CQ 4: What Testing Modalities Are Useful for}

Diagnosing the Degree of Tumor Extension (T-Stage)?

Recommendation

Contrast-enhanced CT and EOB-MRI can be used. If bile duct invasion is suspected, imaging modalities for examining the bile ducts may be useful (strong recommendation).

\section{Description}

According to the staging criteria for ICC in General Rules for the Clinical and Pathological Study of Primary Liver Cancer (revised 6th edition), the T-stage for ICC is defined by the number of tumors, tumor diameter, and the presence of vascular invasion, as is the case with HCC. It is therefore necessary to perform imaging studies to evaluate these parameters.

Grossly, a mass-forming type ICC lesion is a grayishwhite solid tumor with a clear border. However, owing to the absence of a fibrous capsule, tumor borders are often obscured in imaging studies (plain CT, plain MRI, noncontrast-enhanced US). Thus, contrast enhancement is essential. 
Contrast-enhanced CT is widely used for the differential diagnosis and localization of ICC, owing to its simplicity, widespread availability, and reasonable cost [83]. Localization is determined based on the extent of an irregularly shaped hypointense area in the portal and equilibrium phases. EOB-MRI is useful for the intrahepatic localization of ICC because it can visualize the lesion as a well-defined hypointense area in the hepatocellular phase [84]. The Kupffer phase of contrast-enhanced US has been shown to be highly sensitive for detecting ICC because it can visualize the tumor as a clear defect [85]. However, contrast-enhanced US remains an adjunct to contrast-enhanced CT/MRI, owing to blind spots and suboptimal reproducibility. While it is difficult to diagnose periductal-infiltrating ICC on conventional contrast-enhanced CT/MRI, owing to unremarkable Glisson sheath thickening and weak contrast enhancement, diffusion-weighted MRI or PET images may be useful for localization of this ICC type.

Vascular invasion is an important determinant of prognosis, as demonstrated in a meta-analysis (pooled $\mathrm{HR}, 1.87$; 95\% confidence interval (CI), 1.44-2.42) [86], and it is important to accurately determine whether vascular invasion is present or absent. When using multidetector-row CT (MDCT), a modality with high resolution, the sensitivity and specificity are, respectively, $89 \%$ and $92 \%$ for detecting portal vein invasion and $84 \%$ and $93 \%$ for detecting hepatic artery invasion [87]. Multiplanar reconstruction and maximum intensity projection images can also be used to improve visualization and facilitate the evaluation of vascular invasion. In addition, contrast-enhanced MRI is considered to be as effective as CT for evaluating vascular invasion [88]. Because of its high spatial resolution, contrast-enhanced US has been shown to be effective in visualizing vascular invasion at the peripheral level, which cannot be captured by contrast-enhanced CT/MRI, although its superiority remains to be demonstrated.

For ICCs originating near the hilar region, the extent of bile duct invasion needs to be determined in detail, as is the case with hilar cholangiocarcinoma. Endoscopic ultrasound is effective for diagnosing hilar cholangiocarcinoma, especially for identifying cases amenable to surgical resection [89]. Direct cholangiography has good spatial resolution and is useful in determining the extent of bile duct invasion [90]. In addition, mapping biopsy for determining the extent of horizontal extension can subsequently be performed to reduce the incidence of positive resection margins [91]. In a study comparing MDCT and mapping biopsy, the diagnostic accuracy was higher with MDCT (79.7\%) than with mapping biopsy (73.0\%), and this was further improved by combining the two modalities [92]. However, during biopsy under fluoroscopic guidance, the use of forceps is restricted, and it is often difficult to guide the forceps to the target site. Even if the forceps can be guided to the target site, they may slip against the tissue, leading to inadequate tissue collection. In such cases, a cholangioscope can be inserted into the bile duct to perform biopsy under direct vision [93].

\section{CQ 5: What Imaging Modalities Are Useful in}

Detecting Lymph Node Metastasis?

Recommendation

CT, MRI, and FDG-PET are useful for detecting LNM, although their diagnostic accuracy is not necessarily high (strong recommendation).

\section{Description}

The Report of the 20th National Follow-up Survey showed that about $15 \%$ of patients with ICC had LNM at the time of initial diagnosis [8]. Whether a LN detected by CT/MRI is an enlarged reactive node or a metastatic node can be determined based on size, contrast enhancement pattern, and appearance of the margin (extranodal invasion).

In general, the frequency of LNM tends to increase with LN size, and therefore, "node size" is used as a diagnostic criterion for LNM. Enlarged LNs with a shortest diameter of $\geq 1 \mathrm{~cm}$ are commonly regarded as metastatic in clinical practice. However, the diagnostic performance of this size-based criterion is not necessarily high, with $20 \%-61 \%$ sensitivity and $86.4 \%-88 \%$ specificity [94].

Metastatic LNs appear hyperintense on MRI diffusion-weighted images, enabling differentiation between benign and malignant nodes [95]. However, resolution remains an issue because small LNs are still difficult to visualize.

With contrast enhancement, metastatic LNs are visualized as round or oval masses, and heterogeneous and ring-like enhancement patterns inside the LNs are suggestive of metastasis. The diagnostic performance of contrast enhancement patterns is reported to be $50 \%$ sensitivity and $83.3 \%-89 \%$ specificity $[96,97]$.

FDG-PET can detect LNM with 33\%-87.5\% sensitivity and $75.0 \%-92.3 \%$ specificity, showing better diagnostic performance than CT/MRI [94, 97, 98]. However, FDG-PET and PET/CT also have diagnostic limitations - the presence of concomitant cholangitis can result in false positives. 
Extranodal invasion in metastatic LNs can be diagnosed based on the indistinct node borders and "fluffing" in the stroma and is generally positively correlated with node size [99]. However, in cases of highly malignant tumors, extranodal invasion may occur even in relatively small LNs.

Thus, the imaging diagnosis of LNM based on size, contrast enhancement patterns, and extranodal invasion has high specificity but is limited by low sensitivity. The reason for this is that nonmetastatic LNs rarely show imaging abnormalities, and it is difficult to detect small metastatic nodes and micrometastasis that do not show enlargement on imaging. It is worth bearing in mind that the diagnostic accuracy for $\mathrm{N}$ stage is not necessarily high.

\section{CQ 6: What Imaging Modalities Are Useful for}

Detecting Distant Metastasis?

Recommendation

$\mathrm{CT}$ is useful in detecting lung metastasis (strong recommendation). If bone metastasis is suspected, bone scintigraphy or FDG-PET may be useful (strong recommendation).

\section{Description}

According to the Report of the 20th National Followup Survey [8], the incidence of extrahepatic recurrence of ICC is $17.5 \%$ (164/936), and the organs/structures affected by recurrence include LNs (37.8\%), lung (25.0\%), peritoneum (16.5\%), bone (11.6\%), and brain (0.6\%). Among 986 patients diagnosed with ICC between 2010 and 2012 in the Surveillance Epidemiology and End Results program in the USA, distant metastasis occurred in the lung (27.6\%), bone (18.9\%), and (distant) LNs (11.4\%) [100]. This section discusses metastasis of ICC to the lung and bone, the most important organs affected by metastasis.

Chest CT is routinely used to detect lung metastasis [101]. This is because of its relatively short examination time and high spatial resolution. Combining abdominal dynamic contrast-enhanced CT with chest imaging is also useful because it enables both intrahepatic lesions and lung metastasis to be evaluated. By comparison, MRI is considered to be inferior to CT in the diagnosis of pulmonary nodules [101], owing to its slightly lower spatial resolution, longer acquisition time, limited ability to differentiate benign and malignant lung masses based on the rate of increase in signal intensity between noncontrast and contrast-enhanced MRI, and motion artifacts due to breathing. To detect metastasis on FDG-PET, it is essential to compare FDG-PET images with CT images to visually assess the contrast between the lesion and the sur- rounding tissue [102-104]. Standardized uptake value can also be used for semiquantitative assessment. However, care should be taken when interpreting standardized uptake value data because the values for benign and malignant lesions often overlap. Also, the high sensitivity of PET-CT for detecting lesions means that it can detect metastasis that cannot be visualized on other imaging modalities $[79,81]$

Bone destruction patterns observed in bone metastatic lesions are indirect findings indicating osteolysis by osteoclasts, rather than direct invasion by cancer cells. Therefore, morphological diagnosis by X-ray or CT is based on abnormal shadows representing local bone resorption or bone formation [101]. Although MRI is less sensitive than X-ray and CT in detecting signals from the cortical bone, making it more difficult to visualize the destruction of bone structure, diffusion-weighted images are more sensitive for detecting bone metastasis and the degree of lesion extension [101]. Bone scintigraphy and FDG-PET are also useful, especially for their ability to scan the whole body in a single imaging session, whereas $\mathrm{X}$-ray, CT, and MRI can be used for examination only within the scanned range. Note, however, that bone scintigraphy and FDG-PET findings reflect metabolic responses and may lead to false positives due to benign lesions with increased metabolism and false negatives for inactive tumors. FDG-PET can also detect unexpected metastasis or multiple cancers in tissues other than bone, which may lead to a change in treatment strategy $[79,81]$.

\section{CQ 7: In Which Patients Should Tumor Biopsy Be Performed?}

Recommendation

In unresectable cases, tumor biopsy should be considered when deemed necessary for the purposes of differential diagnosis and drug therapy selection (strong recommendation).

\section{Description}

Mass-forming type ICC is characterized by ring-like early enhancement at its margins and delayed enhancement at its center. In typical cases, imaging findings can lead to the final diagnosis. Therefore, pathological scrutiny by tumor biopsy is basically unnecessary, especially in resectable cases. However, some atypical ICCs exhibit early heterogeneous enhancement $[83,105]$ and are difficult to differentiate from HCC. For hepatic masses that have progressed to an unresectable stage, the drug therapy of choice naturally differs depending on the final diagnosis (HCC, ICC, or others). Therefore, when determin- 
ing the drug regimen, it is very important to confirm the histological type of the tumor by biopsy $[1,101,106]$.

Bleeding is an important early complication of needle biopsy. In a study on liver biopsies performed to diagnose hepatic mass lesions [107], complications occurred in $0.5 \%$ of patients $(11 / 2,091)$ and were mostly related to bleeding (intra-abdominal bleeding in 6, intrahepatic hematoma in 1 , and biliary bleeding in 1). A notable late complication is peritoneal dissemination. Although there are no reports of this complication in ICC cases, a meta-analysis of HCC cases showed that the incidence of post-biopsy dissemination was $2.7 \%$ overall or $0.9 \%$ per year [108].

Liver biopsy for hepatic mass lesions has a reported sensitivity of $89.6 \%-97.5 \%$ for HCC and $92.7 \%-95.8 \%$ for metastatic HCC [107] and a low false positive rate (0.08\%), but the reported false negative rate is $6.4 \%$ of cases. Therefore, the possibility of false negatives must be kept in mind when interpreting the pathological results of liver biopsy.

\section{CQ 8: What Types of ICC Are Indicated for Surgical}

Treatment in Terms of Tumor Condition?

Recommendation

A solitary tumor with no LNM is the best indication for hepatectomy. There is no restriction on tumor size (strong recommendation).

\section{Description}

Hepatectomy is the only curative treatment for ICC. According to the General Rules for the Clinical and Pathological Study of Primary Liver Cancer, ICC is classified into mass-forming type, periductal-infiltrating type, and intraductal-growth type [34]. The clinicopathological findings (frequency, site of origin, histopathological features, extension pattern, etc.) and surgical outcomes of ICC differ according to its macroscopic classification [39, 109-114]. The intraductal-growth type is associated with excellent surgical outcomes and thus is a good indication for hepatectomy $[39,111,113,114]$. Periductal-infiltrating type without hilar invasion and without jaundice is also associated with good surgical outcomes [115]. In contrast, the mass-forming type is associated with high recurrence rates even after curative resection, with a 5 -year survival rate of $30 \%-40 \%[39,111,113,114,116-$ 121].

Among mass-forming type ICC lesions, a solitary tumor with no LNM is the best indication for hepatectomy. The number of tumors and the presence or absence of LNM are known to be important prognostic factors for the mass-forming type $[8,38,113,114,116,121-123]$. A solitary tumor without LNM should be treated surgically.

Clinical Practice Guidelines for

Intrahepatic Cholangiocarcinoma
Multiple tumors (including intrahepatic metastases) with LNM are associated with poorest surgical outcomes, and the long-term survival rate is low $[117,124]$. Because of the difficulty of accurately identifying the number of tumors and the presence/absence of LNM before surgery, the indication for surgery should be carefully determined based on various examinations.

The 5-year survival rate decreases with an increasing number of tumors: $46.8 \%$ for a single tumor, $33.6 \%$ for two tumors, and $11.1 \%$ for three tumors [8]. Many other studies have also reported poor 5-year survival rates in cases with multiple tumors [38, 113, 114, 121, 124, 125].

Tumors $\leq 2 \mathrm{~cm}$ in size are associated with favorable 5-year survival (63.4\%) [8]. A report has noted that patients with a tumor measuring $\leq 2 \mathrm{~cm}$ with no LNM (N0), no portal vein invasion, and no bile duct invasion have a $100 \% 5$-year survival rate, and thus, complete cure can be expected [37]. On the other hand, there was no significant difference in the 5-year survival rate according to tumor size: $45.9 \%$ for $2-5 \mathrm{~cm}, 30.7 \%$ for $5-10 \mathrm{~cm}$, and $32.3 \%$ for $>10 \mathrm{~cm}$ [8]. Although the cutoff value of $2 \mathrm{~cm}$ for predicting prognosis needs to be further validated, the indication for resection is not restricted by tumor size.

In terms of the surgical indications for patients with vascular/bile duct invasion, the presence of vascular invasion has been associated with poorer prognosis and, in fact, the presence or absence of portal vein and arterial invasion is included as a criterion for defining the T-stage $[34,37]$. In particular, invasion of the bile ducts in the hilar region (B3, B4), which are the major bile ducts, is associated with poor prognosis and is included as a criterion for the T-stage as well. The presence of vascular/bile duct invasion is associated with poor prognosis but does not indicate unresectable ICC.

About one-third of patients who have undergone surgical resection of ICC have LNM, and the 5-year survival rate is significantly decreased in these patients $[37,38,113]$. A multicenter study conducted by JSHBPS showed that the 5 -year survival rate for patients with positive LNM was 7\%, with only 3 of 139 patients surviving for 5 years after hepatectomy [111]. A subsequent analysis of 419 resected cases conducted by LCSGJ showed that the 5-year survival rate of patients with LNM was 11.1\% [37]. In the Report of the 20th National Follow-up Survey on Primary Liver Cancer (20082009), the 5-year survival rate of patients with LNM (N1) was reported to be $19.4 \%$ [8].

However, another study has shown that long-term survival was achieved in patients with a small number of LNMs [125]. The staging classification used in the multicenter study conducted by JSHBPS [38] and that present- 
ed in the General Rules for the Clinical and Pathological Study of Primary Liver Cancer, 6th edition [34] also classify patients with LNM and T-stage $\leq 3$ as stage IVA, suggesting that resection is indicated for some patients with LNM (who were all classified as stage IVB in the 5th edition of the convention). In fact, the median survival time (MST) of patients classified as T1-T3N1M0 was 16.6 months, which was not significantly different from 22.5 months for those classified as T4N0M0 $(p=0.95)$ [37]. A detailed definition of surgical indications for node-positive cases remains an issue for the future.

Although there are few reports on liver transplantation for ICC, a favorable outcome (5-year recurrence rate, $18 \%$; 5 -year survival rate, $65 \%$ ) was reported in patients with a preoperative diagnosis of HCC who underwent liver transplantation and were postoperatively diagnosed pathologically as having ICC measuring $\leq 2 \mathrm{~cm}$ [126]. Further studies are still needed to address the indication for liver transplantation.

\section{CQ 9: What Are Safe and Reasonable Surgical \\ Techniques?}

Recommendation

The extent of liver resection should be performed to achieve negative surgical margins and sufficient remaining liver function (strong recommendation).

\section{Description}

In patients with ICC, surgical margin status after hepatectomy is a prognostic factor [113, 127-129]. Hepatectomy should be performed to achieve negative surgical margins. For mass-forming ICC, which often associated with chronic hepatitis or cirrhosis, the extent of liver resection should be determined considering remaining liver function, as in the case of HCC. In terms of the extent of liver resection, there was no difference in survival or recurrence-free survival between major hepatectomy (hemihepatectomy or more) and minor hepatectomy (segmentectomy or less), but the postoperative complication rate was considerably higher after major hepatectomy [130]. A study has suggested that small peripheral ICCs with no macroscopic vascular invasion and negative tumor markers are a good indication for laparoscopic partial hepatectomy [131].

\section{CQ 10: Is There Any Significance to Lymph Node}

Dissection?

Recommendation

The significance of LN dissection is currently unclear (no recommendation).

\section{Description}

The incidence of LNM in ICC is reported to be about $30 \%-53 \%$ for the mass-forming type $[39,113,120,132]$ and more than $60 \%$ for the periductal-infiltrating type or mass-forming plus periductal-infiltrating type [39, 113, $117,120,132]$. On the other hand, the intraductal-growth type is rarely associated with LNM $[39,113,117]$. LNM commonly occurs in nodes in the hepatoduodenal ligament, around the common hepatic artery, and behind the pancreatic head. Metastasis to the LNs of the gastric lesser curvature can also occur in patients with ICC in the left liver.

The significance of prophylactic LN dissection is not clear in cases where no LNM was detected on preoperative imaging and laparotomic exploration. Among patients with a solitary tumor without LNM, there was no difference in the outcome between those who underwent LN dissection and those who did not [119]. A retrospective study showed that LN dissection was more effective when $\geq 3$ nodes were dissected than when $<3$ nodes were dissected [133]. A collaborative Korea-Japan study revealed that the number of LNMs $(0,1-3, \geq 4)$ was associated with poor prognosis, and surgical retrieval of $\mathrm{LN} \geq 4$ was associated with improved survival outcome in ICC patients with LNM, though LN dissection did not improve survival outcome in patients with LNM [134]. Moreover, there is an international consensus conference recommendation that LN dissection be considered in the surgical treatment of ICC [101]. Some also argue that the results of LN dissection can be used to guide staging and determine the indication for adjuvant chemotherapy $[101,135]$. Meanwhile, others argue that LN dissection should be limited and performed only for obtaining samples for staging and other purposes [136-139]. Prophylactic LN dissection in patients with concomitant cirrhosis should be performed with care because it is associated with a significant increase in postoperative complications [140].

Some recent studies, although retrospective, have demonstrated the prognostic value of LN dissection using new methods of analysis [141-143]. Future studies are needed to determine the significance of $\mathrm{LN}$ dissection.

\section{CQ 11: What Are the Indications of Percutaneous} Ablation Therapy?

Recommendation

Percutaneous ablation therapy may be considered for patients with ICC who are ineligible for surgical resection or chemotherapy, owing to deteriorated hepatic functional reserve or comorbidities (weak recommendation). 


\section{Description}

For patients with ICC who have normal hepatic functional reserve but are ineligible for surgical resection owing to tumor extension, chemotherapy is the standard treatment. On the other hand, there are cases who cannot receive hepatic resection or chemotherapy, owing to comorbidities or deteriorated hepatic functional reserve. In these cases, percutaneous ablation therapy may be considered [144, 145].

Kolarich et al. [146] reported that survival was significantly better in patients with UICC stage I ICC (single tumor, no vascular invasion) who underwent radiofrequency ablation (RFA) compared with no treatment (2.1 years vs. 0.7 years, $p=0.012$ ). Another study [147] suggested that percutaneous ablation therapy could be compatible with repeat hepatectomy for recurrent ICC measuring less than $3 \mathrm{~cm}$. However, percutaneous ablation therapy was performed as initial treatment for ICC in only $3.6 \%$ according to the Report of the 20th Japanese National Follow-up Survey [8]. In the SEER database from the USA, only $5.2 \%$ of ICC patients received percutaneous ablation therapy alone [145]. Therefore, percutaneous ablation therapy is limited in the clinical practice.

RFA was used in most reports on percutaneous ablation therapy, and there are even fewer reports on microwave ablation. Therefore, RFA may be the first choice of percutaneous ablation therapy for ICC that is ineligible for resection.

\section{CQ 12: What Drug Therapies Are Recommended for Unresectable ICC?}

Recommendation

The recommended drug therapies for unresectable ICC are gemcitabine + cisplatin $+\mathrm{S}-1$, gemcitabine + cisplatin, and gemcitabine $+\mathrm{S}-1$ combination therapies (strong recommendation).

\section{Description}

Conventional chemotherapy regimens have been developed for biliary tract cancer (BTC) as a whole, but no chemotherapy has been developed specifically for ICC. In the UK, a phase III study (ABC-02) [148] was conducted to compare gemcitabine alone with gemcitabine plus cisplatin (GC) for unresectable BTC. MST was significantly better in the GC group $(n=204)$ than in the gemcitabine monotherapy group $(n=206)$ (MST, 11.7 vs. 8.1 months; HR, 0.64; 95\% CI, 0.52-0.80). Based on these results, GC therapy has become a standard treatment for unresectable BTC. In the ABC-02 study, ICC accounted for 80 of 410 patients. Similar results were reported from a Japa-

Clinical Practice Guidelines for

Intrahepatic Cholangiocarcinoma nese phase II study in 84 patients with unresectable BTC (BT22) [149], with MST of 11.2 versus 7.7 months (HR, $0.69 ; 95 \%$ CI, 0.42-1.13). In the BT22 study, ICC accounted for 28 patients. In a meta-analysis of data from the ABC-02 and BT22 studies [150], a subgroup analysis of ICC also showed a trend toward the superiority of GC over gemcitabine alone in terms of MST (HR, 0.54; 95\% CI, 0.36-0.81).

The Japan Clinical Oncology Group (JCOG) conducted a phase III trial to evaluate the noninferiority and superiority of gemcitabine + S- 1 combination therapy (GS) compared with GC therapy (JCOG1113/FUGA-BT study; UMIN000010667) [151]. The results demonstrated noninferiority but not superiority of GS therapy compared with GC therapy (MST, 15.1 vs. 13.4 months; HR, 0.945; 90\% CI, 0.777-1.149). In the JCOG1113 study (FUGABT), ICC accounted for 94 of 354 patients. There was no substantial difference in the response rate or adverse events (AEs) between the GC and GS groups. At the same time, both cisplatin and S-1 require dose reduction in patients with renal dysfunction, so the presence or absence of renal dysfunction does not guide treatment selection. Based on these results, GS therapy has become an option for unresectable BTC.

Recently, favorable overall survival was demonstrated in a phase II trial of nab-paclitaxel plus gemcitabine-cisplatin for advanced BTC [152], and randomized controlled trials have investigated treatment of BTC with gemcitabine in combination with oxaliplatin and several other regimens [153-161]. However, no treatment to date has demonstrated a significant survival benefit compared with GC therapy in phase III trials [153-161].

The Kansai Hepatobiliary Oncology Group (KHBO) conducted a phase III trial to evaluate the superiority of gemcitabine + cisplatin + S-1 combination therapy (GCS) over GC therapy in terms of MST (KHBO1401MIT-SUBA study; NCT02182778) [162]. The results demonstrated superiority of GCS therapy over GC therapy (MST, 13.5 vs. 12.6 months; HR, 0.79; 90\% CI, 0.628-0.996), and GCS has become a new standard treatment for unresectable BTC. In the study, ICC accounted for 78 of 246 patients. GCS therapy achieved a higher response rate of $41.5 \%$, compared with $15.0 \%$ with GC therapy. In terms of the incidence of grade $\geq 3$ AEs according to the NCI-CTC (version 4.0), stomatitis, diarrhea, and skin rash were significantly more common in the GCS group, and peripheral neuropathy was more common in the GC group. Thus, the recommended (first-line) chemotherapy regimens for unresectable ICC are gemcitabine + cisplatin + S-1, gem- 
citabine + cisplatin, and gemcitabine + S-1 combination therapies.

Evidence is currently lacking on recommended second-line treatments for ICC after failure of standard treatment. In Japan, S-1 has been approved for treatment of unresectable BTC and has a reported response rate of $4.0 \%-22.7 \%$ for gemcitabine-refractory BTC [163-165].

In Japan, the antihuman PD- 1 antibody pembrolizumab has been approved for an additional indication of advanced/recurrent solid tumors with high microsatellite instability that have progressed after anticancer chemotherapy. In a phase II trial of pembrolizumab monotherapy for such high microsatellite instability solid tumors, 86 patients with 12 types of cancer were enrolled, including 4 with cholangiocarcinoma and 4 with ampullary carcinoma, and the following results were reported: MST was not reached, the 2 -year survival rate was $64 \%$, and the response rate was 58\% [166]. Complete response was achieved in 1 of 4 patients with cholangiocarcinoma. Thus, pembrolizumab is recommended only when the standard treatment cannot be used.

An integrated analysis of phase I and II studies of the $T R K$ inhibitor entrectinib in patients with solid tumors harboring neurotrophic tyrosine receptor kinase (NTRK) fusion genes included 10 organs and 19 histological types in 54 patients, including 1 patient with ICC; MST was 21 months, duration of response was 10 months, progression-free survival was 11 months, and response rate was $57 \%$, and 4 patients achieved complete response [167].

Entrectinib was approved in Japan for national health insurance-covered treatment of advanced/recurrent NTRK fusion gene-positive solid tumors in June 2019. Although the prevalence of NTRK fusion gene expression in ICC is extremely low, as evidenced by a report that only 1 of 28 ICC patients was positive for NTRK fusion genes [168], treatment with entrectinib can be considered because it is expected to be effective in situations where the standard treatment is unlikely to be effective. In July 2021, larotrectinib was approved in Japan for national health insurance-covered treatment of patients with TRK fusion-positive solid tumors.

Pemigatinib was recently approved in Japan as a national health insurance-covered treatment for unresectable BTC with fibroblast growth factor receptor 2 fusions/ rearrangements. An international multicenter phase II study (FIGHT-202) demonstrated that MST was 21 months, duration of response was 7.5 months, progression-free survival was 6.9 months, response rate was $35.5 \%$, and the disease control rate was $82.2 \%$ [169]. Fibroblast growth factor receptor 2 fusions/rearrangements have been detected in $5.3 \%$ to $13.6 \%$ of patients with ICC [170-172].

Isocitrate dehydrogenase gene mutations can cause intracellular DNA methylation and promote tumor formation. A phase III clinical study evaluating the role of ivosidenib for BTC, which is a targeted mutant isocitrate dehydrogenase inhibitor, showed better progression-free survival of 2.7 months [173]. However, as of this writing, this treatment is not covered by national health insurance in Japan.

\section{CQ 13: Is Neoadjuvant Chemotherapy Recommended?} Recommendation

There is no evidence for a benefit of neoadjuvant chemotherapy (no recommendation).

\section{Description}

There are two types of neoadjuvant chemotherapy: one used to improve the outcome of surgical resection in resectable cases (neoadjuvant chemotherapy in the narrow sense) and the other used in unresectable cases with the aim of possible response to chemotherapy and subsequent conversion to surgical resection (neoadjuvant chemotherapy in the broad sense). There is no evidence for neoadjuvant chemotherapy in resectable cases. However, to further improve treatment outcomes, neoadjuvant chemotherapy should be considered in cases with LNM or multiple tumors, which are associated with poor resection outcomes. For such evaluations, well-documented prognostic factors, such as the presence of LMN, intrahepatic metastasis and/or vascular invasion, and tumor markers, should be included as inclusion criteria [174]. The inclusion of resectable cases leaves the possibility that cases become unresectable after neoadjuvant chemotherapy. Thus, the first step will be to conduct clinical studies with safety and the curative resection rate as endpoints.

Administration of chemotherapy in patients with unresectable disease has been reported to result in reductions in tumor size and vascular invasion and the disappearance of abnormal uptake in regional and distant LNs on FDG-PET, followed by conversion to resectable disease $[175,176]$. In Japan, the conversion rate after chemotherapy (gemcitabine monotherapy) for unresectable BTC, including ICC, was reported to be $36.4 \%$ in a retrospective analysis [177]. The reasons for not performing initial resection in patients with ICC were extensive vascular invasion in 3 patients and insufficient expected residual liver volume after resection in 1 patient. A recent study of ICC alone showed a conversion rate of 53\% (39/74 patients); MST was 24.1 months in converted pa- 
tients, compared with 25.7 months in patients with an initially resectable tumor $(n=82 ; p=0.391)$. Moreover, there was no significant difference in recurrence-free survival between the two groups [178]. These reports suggest the effectiveness of chemotherapy for unresectable ICC, but the studies were retrospective in nature and did not clarify the significance of neoadjuvant chemotherapy.

\section{CQ 14: Is Adjuvant Chemotherapy Recommended?}

Recommendation

Adjuvant chemotherapy may be considered because some regimens have demonstrated tolerability and suggested efficacy (weak recommendation).

\section{Description}

Most studies on adjuvant therapy for ICC are retrospective in nature and can be roughly divided into two groups: studies on BTC, which includes extrahepatic cholangiocarcinoma and gallbladder cancer, and studies including ICC only. Most of the studies on BTC do not include stratified analysis of ICC.

For adjuvant chemotherapy, gemcitabine-based therapies are the most commonly used, followed by fluoropyrimidine-based therapies. Because most of the reported studies are retrospective and there is controversy regarding their survival benefit, it is difficult to draw a conclusion on the effectiveness of these therapies even after reviewing reports that have been adjusted for selection bias and other factors $[179,180]$. Although a systematic review of retrospective studies [181] suggested the effectiveness of adjuvant chemotherapy, the analysis was not focused on ICC, and the included studies appeared to be biased in terms of background factors. Further, a metaanalysis has found that adjuvant chemotherapy is associated with improved overall survival and should be offered to ICC patients after surgery, especially those with advanced disease [182]. However, the clinical benefit of adjuvant chemotherapy for advanced ICC patients remains unclear. Therefore, the evidence for the clinical benefit of adjuvant chemotherapy was assessed as weak.

Some prospective studies have evaluated the tolerability and pharmacokinetics of adjuvant chemotherapy [183188]. The regimens evaluated were gemcitabine alone, S-1 alone, and GC. Although the need for dose reduction has been suggested for some adjuvant chemotherapy regimens, most regimens have been shown to be well tolerated. In prospective comparative studies, ICC was studied not exclusively but as a form of BTC [189-194]. In a clinical study designed to examine whether adjuvant chemotherapy with capecitabine alone would prolong survival compared with

Clinical Practice Guidelines for

Intrahepatic Cholangiocarcinoma surgery alone in patients with BTC (BILCAP study), no survival benefit was demonstrated in the intention-to-treat population, but there was survival benefit with an HR of 0.75 in theper protocol population [192]. Thus, capecitabine monotherapy has the potential to become a standard adjuvant chemotherapy for BTC and is also recommended in overseas guidelines [195]. However, in a subgroup analysis, capecitabine did not show a significant survival benefit in patients with ICC. Further, as of this writing, this therapy is not covered by national health insurance in Japan. Subsequently, in a clinical study designed to examine whether gemcitabine + oxaliplatin combination therapy would prolong recurrence-free survival compared with surgery alone (PRODIGE 12-ACCORD 18 study), the superiority of the combination therapy was not demonstrated [193]. In the study, about $45 \%$ of patients had ICC, and even stratified analysis showed no superiority of the adjuvant chemotherapy. In another prospective comparative study (KHBO1208), gemcitabine monotherapy was compared with S-1 monotherapy, although it was a phase II trial for BTC including ICC (40\%) [194]. The HR for overall survival with S-1 monotherapy was 0.477 compared with gemcitabine monotherapy $(p=0.0606)$.

Efficacy was not demonstrated in intention-to-treat analyses in the following studies: a study conducted by Takeda et al. [189] to evaluate the efficacy of mitomycin $\mathrm{C}+5$-FU in BTC, including cholangiocarcinoma; the ESPAC-3 study designed to evaluate the efficacy of 5-FU + folinic acid versus gemcitabine alone in periampullary cancer, mainly extrahepatic cholangiocarcinoma and ampullary carcinoma [190]; and the BCAT study designed to evaluate the efficacy of gemcitabine in extrahepatic cholangiocarcinoma [191]. However, these studies did not include ICC, and therefore, the results should be interpreted with caution.

Taken together, the most reliable evidence is that adjuvant chemotherapy with capecitabine alone demonstrated a survival benefit in BTC, including ICC, in only the per protocol analysis of a prospective comparative study and that the effectiveness of adjuvant chemotherapy with gemcitabine + oxaliplatin was not demonstrated. There was no high-quality evidence for the selection of patients who should receive adjuvant chemotherapy.

Ongoing clinical studies include ASCOT, which compares adjuvant S-1 monotherapy with surgery alone [196], and ACTICCA-1, which compares adjuvant GC therapy with surgery alone [197]. These data clearly demonstrate that adjuvant chemotherapy is well tolerated in ICC, and some studies, including prospective comparative studies, have demonstrated a survival benefit of ad- 
juvant chemotherapy. Therefore, the use of adjuvant chemotherapy may be considered in the treatment of ICC.

\section{CQ 15: Is Stereotactic Radiotherapy Recommended for}

Unresectable ICC?

Recommendation

Stereotactic radiotherapy may be considered for unresectable ICC with tumor diameter $\leq 5 \mathrm{~cm}$ in the absence of metastasis (weak recommendation).

\section{Description}

Chemotherapy is recommended for unresectable ICC. Although no prospective comparative studies have shown the superiority of adding radiotherapy to chemotherapy, radiotherapy is a local treatment option for patients without distant metastasis and those with symptoms associated with local lesions. Stereotactic radiotherapy is an irradiation method that can increase the antitumor effect while reducing AEs by delivering the radiation dose from multiple directions to the lesion only, thereby reducing the radiation dose to the healthy liver. This technique is gaining increasing attention with the advancement of image-guided radiotherapy and respiratory motion management techniques [198].

Most previous studies on stereotactic radiotherapy for ICC have shown a local control rate of around $80 \%$, although they were all phase I/II trials or retrospective studies with a small sample size [198-203]. Some studies have found tumor response increased with increasing radiation dose, with a better response obtained when the biologically equivalent dose exceeded $80-100 \mathrm{~Gy}$ (when $\alpha / \beta$ $=10$, equivalent to $67-83$ Gy with conventional fractionated irradiation at $2 \mathrm{~Gy} /$ fraction) $[200,201]$. Another report found that the smaller the tumor diameter, the better the local control rate. It should be noted that in Japan, stereotactic radiotherapy for primary liver cancer is covered by national health insurance only for lesions $\leq 5 \mathrm{~cm}$ in diameter in the absence of metastasis. Moreover, based on evidence in the treatment of HCC and metastatic liver tumors, stereotactic radiotherapy for larger tumors measuring $>5 \mathrm{~cm}$ is often associated with increased risk of compromised residual liver function after treatment. It is therefore reasonable to consider particle radiotherapy in such cases.

\section{CQ 16: Is Particle Radiotherapy Recommended for} Unresectable ICC?

Recommendation

Particle radiotherapy may be considered for unresectable ICC without metastasis (weak recommendation).

\section{Description}

Although chemotherapy is recommended for unresectable ICC, its therapeutic effect is not satisfactory, and further development of chemotherapy agents and effective local therapies are needed. Although all previous studies on particle radiotherapy for unresectable ICC were phase I/II trials or retrospective studies with small sample sizes, favorable local control rates have been reported regardless of tumor size, and long-term survival has also been achieved in some cases [204-208]. While there is no established dose-fractionation method or total dose for particle radiotherapy that can be recommended on scientific grounds, many reports have described the use of higher doses than in photon radiotherapy, with better local control rates in higher dose groups [207]. Gastrointestinal morbidities and cholangitis have been reported as AEs, but the incidence of serious AEs is low [204-208].

Although further studies with a higher level of evidence are needed, particle radiotherapy is currently a promising treatment option for ICC because it can be generally performed safely even for large tumors. As of 2020 , particle radiotherapy for ICC is performed as advanced medical care in Japan.

\section{Clinical Topic}

\section{Clinical Topics 1}

Is it possible to distinguish between hilar cholangiocarcinoma and ICC involving the hepatic hilum?

\section{Description}

Pathological identification of the primary site of a tumor is one of the basic principles of pathological diagnosis. Currently, however, there is still a need to distinguish between hilar cholangiocarcinoma and ICC involving the hepatic hilum $[40,209,210]$. In fact, clinically, the prognosis and surgical treatment of tumors arising in the hilar region are almost the same as those of ICC involving the hepatic hilum. Moreover, there is currently no established immunohistological method for differentiating these primary sites pathologically.

However, for hilar tumors, it is possible to differentiate between hilar cholangioarcinoma and ICC involving the hepatic hilum by referring to the following: (1) careful sectioning, (2) location of stenosis, (3) pathological evaluation of tumor volume and invasion, (4) the presence of BilIN, (5) confirmation of the elastic fibers surrounding the hilar region (Elastica van Gieson staining) [211], and 
Fig. 3. Example of distinguishing between hilar cholangiocarcinoma and ICC involving the hilum. The appearance of tumor growth suggests hilar cholangiocarcinoma (upper panel). On histological examination of the specimen shown in the red box, however, although not clear on the hematoxylin and eosin section (bottom left), the Elastica van Gieson staining (bottom right) shows that the tumor is located outside the elastic fibers (red arrowhead). This indicates that it is located in the liver parenchyma (yellow arrowhead), leading to a diagnosis of ICC involving the hilum. ICC, intrahepatic cholangiocarcinoma (adapted from Reference [202]).

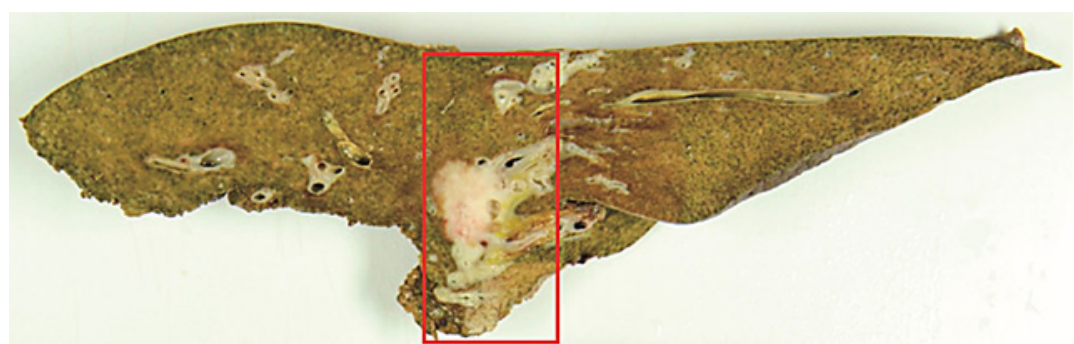

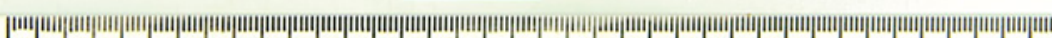
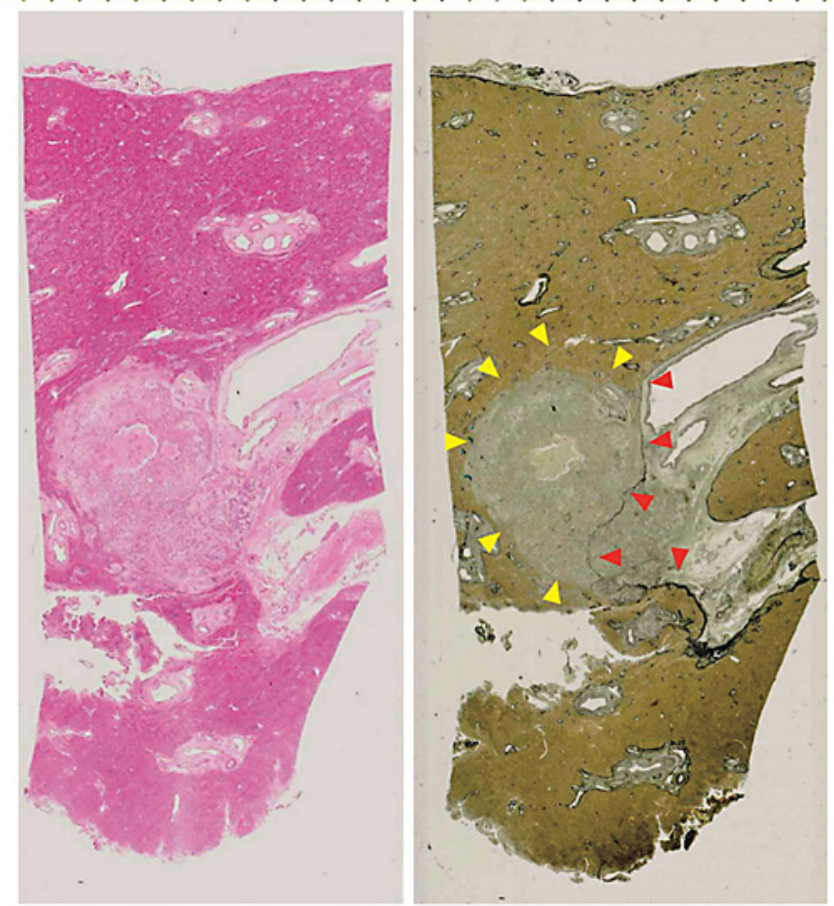

(6) clinical imaging findings (Fig. 3). This method of pathological differentiation is also used to determine whether a tumor originated near the intrapancreatic bile duct or the biliary tract.

The liver and extrahepatic bile ducts are embryologically distinct. In recent years, clinicopathological studies of BTC have been conducted not only on morphological aspects but also on genetic abnormalities and driver molecular pathways. Furthermore, molecular targeted therapies have been developed based on the results of these studies. Recently, 32 important driver genes that could be targets of new molecular targeted therapies have been identified and reported to have genetic characteristics that vary according to the site of origin (Fig. 4) [212]. Therefore, it is possible to pathologically distinguish between hilar cholangiocarcinoma and ICC involving the hepatic hilum, and it is necessary to pathologically identify the primary organ.

Clinical Practice Guidelines for

Intrahepatic Cholangiocarcinoma

\section{Acknowledgments}

The authors thank Drs. Shunichi Ariizumi, Hidenori Ojima, Shun Kaneko, Shogo Kobayashi, Yasunori Sato, Kei Shibuya, Hideaki Sueoka, Katsutoshi Sugimoto, Yutaka Suzuki, Ryosuke Takenaka, Yoshiki Naito, Yasunari Fujinaga, Yutaka Midorikawa, and Yasunori Minami for their great contributions to the guidelines.

\section{Conflict of Interest Statement}

The authors have no conflicts of interest to declare. Kudo M. is the editor-in-chief of Liver Cancer. Izumi N., Kokudo N., and Sakamoto M. are associate editors of Liver Cancer. Hatano E. is a member of the Editorial Board of Liver Cancer.

\section{Funding Sources}

The authors have no funding source.

Liver Cancer 2022;11:290-314 


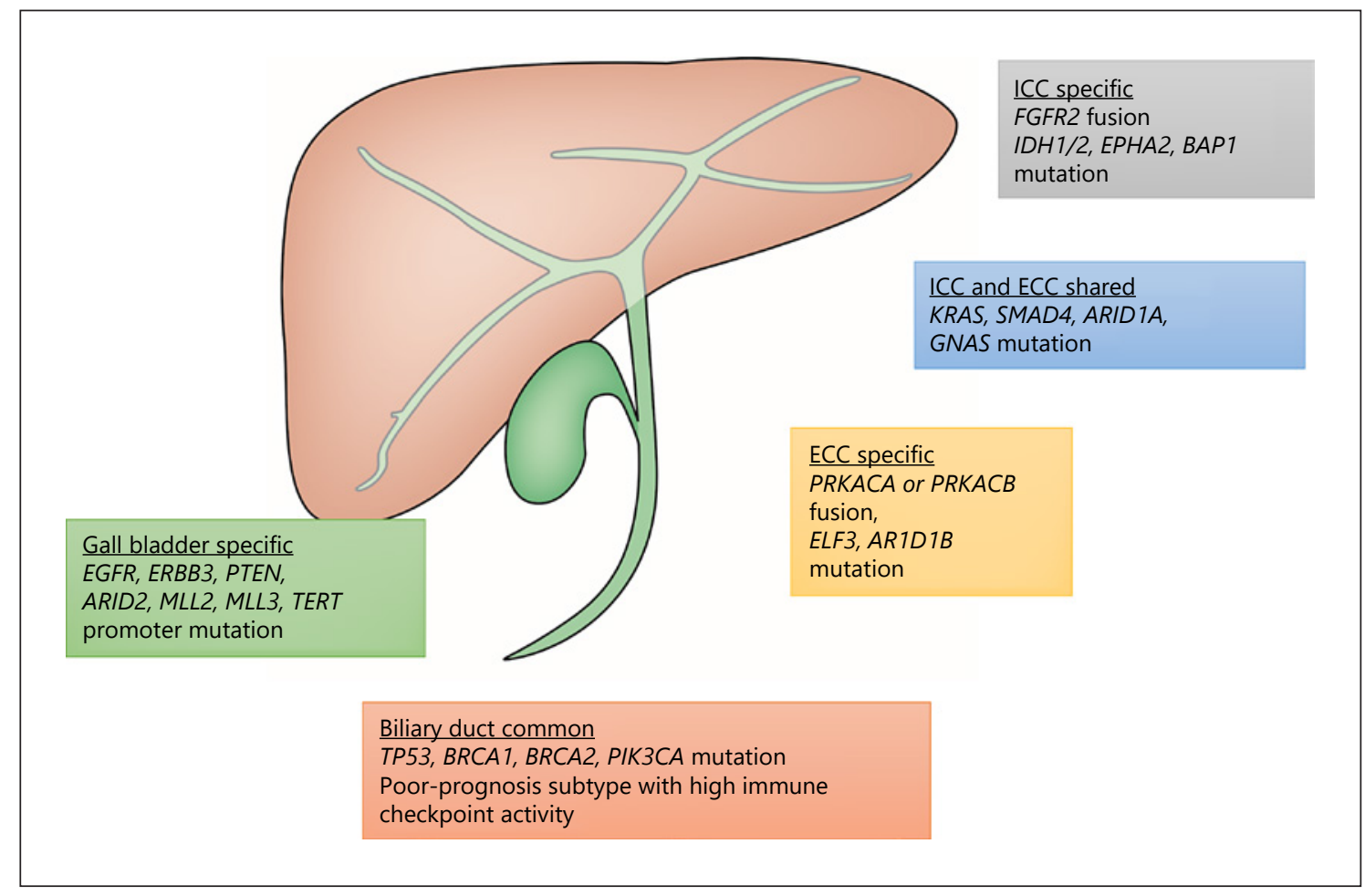

Fig. 4. Locations of origin and genetic abnormalities of biliary carcinomas. ECC, extrahepatic cholangiocarcinoma; ICC, intrahepatic cholangiocarcinoma (adapted and modified from Abe et al. [206]).

\section{Author Contributions}

Kubo: conception and design; Shinkawa, Asaoka, Ioka, Igaki, Izumi, Itoi, Unno, Otsuka, Okusaka, Kadoya, Kudo, Kumada, Kokudo, Sakamoto, Sakamoto, Sakurai, Takayama, Nakashima, Nagata, Hatano, Harada, Murakami, and Yamamoto: drafting of the manuscript; Kubo: critical revision of the manuscript. All the authors approved the final version of the manuscript.

\section{Data Availability Statement}

All data generated or analyzed during this study are included in this article. Further inquiries can be directed to the corresponding author.

\section{References}

1 Bridgewater J, Galle PR, Khan SA, Llovet JM, Park JW, Patel T, et al. Guidelines for the diagnosis and management of intra- hepatic cholangiocarcinoma. J Hepatol. 2014;60: 1268-89.

2 Lee TY, Lin JT, Kuo KN, Wu MS, Ho HJ, Chen TT, et al. A nationwide populationbased study shows increasing incidence of cholangiocarcinoma. Hepatol Int. 2013;7: 226-32.

3 Kim BW, Oh CM, Choi HY, Park JW, Cho H, Ki M. Incidence and overall survival of biliary tract cancers in South Korea from 2006 to 2015: using the National health information database. Gut Liver. 2019;13:104-13.

4 Saha SK, Zhu AX, Fuchs CS, Brooks GA. Forty-year trends in cholangiocarcinoma inci- dence in the US: intrahepatic disease on the rise. Oncologist. 2016;21:594-9.

5 Witjes CD, Karim-Kos HE, Visser O, de Vries E, IJzermans JNM, de Man RA, et al. Intrahepatic cholangiocarcinoma in a low endemic area: rising incidence and improved survival. HPB. 2012;14:777-81.

6 Flemming JA, Zhang-Salomons J, Nanji S, Booth CM. Increased incidence but improved median over all survival for biliary tract cancers diagnosed in Ontario from 1994 to 2012: a population-based study. Cancer. 2016;122: 2534-43.

7 Petrick JL, Braunlin M, Laversanne M, Valery PC, Bray F, McGlynn KA. International trends in liver cancer incidence, overall and by histologic subtype, 1978-2007. Int J Cancer. 2016;139:1534-45.
8 Utada M, Ohno Y, Tamaki T, Sobue T, Endo G. Long-term trends in incidence and mortality of intrahepatic and extrahepatic bile duct cancer in Japan. J Epidemiol. 2014;24:193-9.

9 Website of the Japanese Ministry of Health, Labour and Welfare. Demographic Statistics (in Japanese); 2020. Available from: https:// $\mathrm{w} w \mathrm{w} \cdot \mathrm{m} \mathrm{hl} \mathrm{w} \cdot \mathrm{go} \cdot \mathrm{j} \mathrm{p} / \mathrm{s} \mathrm{t} \mathrm{f} /$ shingi/2r9852000002 njbj-att/ 2r9852000002njg7.pdf

10 Kudo M, Izumi N, Kubo S, Kokudo N, Sakamoto M, Shiina S, et al. Report of the 20th Nationwide follow-up survey of primary liver cancer in Japan. Hepatol Res. 2020;50:15-46. 
11 Forman D, Bray F, Brewster DH, Gombe Mbalawa C, Kohler B, Piñeros M, et al. editors. Cancer incidence in five continents. Lyon; Geneva: International Agency for Research on Cancer; Distributed by WHO Press, World Health Organization; 2014.

12 Ojima $\mathrm{H}$. The epidemiology of cholangiocarcinoma. Kan.Tan.Sui. 2008;57:9-17.

13 Shaib Y, El-Serag HB. The epidemiology of cholangiocarcinoma. Semin Liver Dis. 2004; 24:115-25.

14 Zhang H, Zhu B, Zhang H, Liang J, Zeng W. HBV infection status and the risk of cholangiocarcinoma in Asia: a meta-analysis. Biomed Res Int. 2016;2016:3417976.

15 Kobayashi M, Ikeda K, Saitoh S, Suzuki F, Tsubota A, Suzuki Y, et al. Incidence of primary cholangiocellular carcinoma of the liver in Japanese patients with hepatitis C virusrelated cirrhosis. Cancer. 2000;88:2471-7.

16 Yamamoto S, Kubo S, Hai S, Uenishi T, Yamamoto $T$, Shuto $T$, et al. Hepatitis $C$ virus infection as a likely etiology of intrahepatic cholangiocarcinoma. Cancer Sci. 2004;95: 592-5.

17 Li H, Hu B, Zhou ZQ, Guan J, Zhang ZY, Zhou GW. Hepatitis $C$ virus infection and the risk of intrahepatic cholangiocarcinoma and extrahepatic cholangiocarcinoma: evidence from a systematic review and meta analysis of 16 case-control studies. World J Surg Oncol. 2015; $13: 161$.

18 Palmer WC, Patel T. Are common factors involved in the pathogenesis of primary liver cancers? A meta-analysis of risk factors for intrahepatic cholangiocarcinoma. J Hepatol. 2012;57:69-76.

19 Petrick JL, Campbell PT, Koshiol J, Thistle JE, Andreotti G, Beane-Freeman LE, et al. Tobacco, alcohol use and risk of hepatocellular carcinoma and intrahepatic cholangiocarcinoma: the liver cancer pooling project. Br J Cancer. 2018;118:1005-12.

20 Wongjarupong N, Assavapongpaiboon B, Susantitaphong $\mathrm{P}$, Cheungpasitporn $\mathrm{W}$, Treeprasertsuk S, Rerknimitr R, et al. Non-alcoholic fatty liver disease as a risk factor for cholangiocarcinoma: a systematic review and meta-analysis. BMC Gastroenterol. 2017;17: 149.

21 Shin HR, Oh JK, Lim MK, Shin A, Kong HJ, Jung KW, et al. Descriptive epidemiology of cholangiocarcinoma and clonorchia sis in Korea. J Korean Med Sci. 2010;25:1011-6.

22 Kamsa-ard S, Kamsa-ard S, Luvira V, Suwanrungruang $\mathrm{K}$, Vatanasapt $\mathrm{P}$, Wiangnon $\mathrm{S}$. Risk factors for cholangiocarcinoma in Thailand: a systematic review and meta-analysis. Asian Pac J Cancer Prev. 2018;19:605-14.

23 Lee CH, Chang CJ, Lin YJ, Yeh CN, Chen MF, Hsieh SY. Viral hepatitis: associated intrahepatic cholangiocarcinoma shares common disease processes with hepatocellular carcinoma. Br J Cancer. 2009;100:1765-70.
24 Lee TY, Lee SS, Jung SW, Jeon SH, Yun SC, $\mathrm{Oh} \mathrm{HC}$, et al. Hepatitis B virus infection and intrahepatic cholangiocarcinoma in Korea: a case-control study. Am J Gastroenterol. 2008; 103:1716-20.

25 Kubo S, Kinoshita H, Hirohashi K, et al. Hepatolithiasis associated with cholangiocarcinoma. World J Surg. 1995;19:637-41.

26 Suzuki Y, Mori T, Yokoyama M, Hamba H. Hepatolithiasis: analysis of Japanese nationwide surveys over a period of 40 years. J Hepatobiliary Pancreat Sci. 2014;21:617-22.

27 Ali AH, Tabibian JH, Nasser-Ghodsi N, Lennon RJ, DeLeon T, Borad MJ, et al. Surveillance for hepatobiliary cancers in patients with primary sclerosing cholangitis. Hepatology. 2018;67:2338-51.

28 Shaib YH, El-Serag HB, Davila JA, Morgan R, McGlynn KA. Risk factors of intrahepatic cholangiocarcinoma in the United States: a case-control study. Gastroenterology. 2005; 128:620-6.

29 Petrick JL, Yang B, Altekruse SF, Van Dyke AL, Koshiol J, Graubard BI, et al. Risk factors for intrahepatic and extrahepatic cholangiocarcinoma in the United States: a populationbased study in SEER-Medicare. PLoS One. 2017;12:e0186643.

30 Sharp GB. The relationship between internally deposited alpha-particle radiation and subsite-specific liver cancer and liver cirrhosis: an analysis of published data. J Radiat Res. 2002; 43:371-80.

31 Kubo S, Nakanuma Y, Takemura S, Sakata C, Urata Y, Nozawa A, et al. Case series of $17 \mathrm{pa}-$ tients with cholangiocarcinoma among young adult workers of a printing company in Japan. J Hepatobiliary Pancreat Sci. 2014;21: $479-88$.

32 Mimaki S, Totsuka Y, Suzuki Y, Nakai C, Goto M, Kojima M, et al. Hypermutation and unique mutational signatures of occupational cholangiocarcinoma in printing workers exposed to haloalkanes. Carcinogenesis. 2016; $37: 817-26$.

33 Sobue T, Utada M, Makiuchi T, Ohno Y, Uehara S, Hayashi T, et al. Risk of bile duct cancer among printing workers exposed to 1,2-dichloropropane and/or dichloromethane. J Occup Health. 2015;57:230-6.

34 Liver Cancer Study Group of Japan. General rules for the clinical and pathological study of primary liver cancer. 6th ed. Kanehara \& Co. Tokyo; 2019. p. 27.

35 Brierly JD, Gospodarowicz MK, Wittekind C, editors. ICD-O-3 C22.1 Intrahepatic bile ducts. TNM Classification of Malignant Tumours. 8th ed. Oxford: Wiley Blackwell; 2017. p. 83-4.

36 Sakamoto Y, Kokudo N, Matsuyama Y, Sakamoto M, Izumi N, Kadoya M, et al. Proposal of a new staging system for intrahepatic cholangiocarcinoma: analysis of surgical patients from a nationwide survey of the liver cancer study group of Japan. Cancer. 2016;122:6170.
37 Liver Cancer Study Group of Japan. General rules for the clinical and pathological study of primary liver cancer. 5th ed. Kanehara \& Co. Tokyo; 2008. p. 25.

38 Yamamoto M, Takasaki K, Yoshikawa T, Ueno K, Nakuno M. Does gross appearance indicate prognosis in intrahepatic cholangiocarcinoma? J Surg Oncol. 1998;69:162-7.

39 Uenishi T, Ariizumi S, Aoki T, Ebata T, Ohtsuka M, Tanaka E, et al. Proposal of a new staging system for mass-forming intra- hepatic cholangiocarcinoma: a multicenter analysis by the study group for hepatic surgery of the Japanese society of hepato-biliary-pancreatic surgery. J Hepatobiliary Pancreat Sci. 2014; 21:499-508.

40 Bosman FT, Carneiro F, Hruban RH, Theise $\mathrm{ND}$, editors. WHO classification of tumours of the digestive system. 4th ed. Lyon: IARC; 2010.

41 WHO Classification of Tumours Editorial Board. Digestive system tumours. WHO classification of tumours. 5th ed. Lyon: IARC; 2019.

42 Liver Cancer Study Group of Japan. Classification of primary liver cancer (sixth). Tokyo: Liver Cancer Study Group of Japan, Kanehara \& Co., Ltd; 2019.

43 Zen Y, Adsay NV, Bardadin K, Colombari R, Ferrell L, Haga H, et al. Biliary intraepithelial neoplasia: an international interobserver agreement study and proposal for diagnostic criteria. Mod Pathol. 2007;20:701-9.

44 Kubota K, Nakanuma Y, Kondo F, Hachiya H, Miyazaki M, Nagino M, et al. Clinicopathological features and prognosis of mucin-producing bile duct tumor and mucinous cystic tumor of the liver: a multi-institutional study by the Japan biliary association. J Hepatobiliary Pancreat Sci. 2014;21:176-85.

45 Nakanuma Y, Jang KT, Fukushima N, Furukawa T, Hong SM, Kim H, et al. A statement by the Japan-Korea expert pathologists for future clinicopathological and molecular analyses toward consensus building of intra- ductal papillary neoplasm of the bile duct through several opinions at the present stage. J Hepatobiliary Pancreat Sci. 2018;25:181-7.

46 Lazaridis KN, Gores GJ. Primary sclerosing cholangitis and cholangiocarcinoma. Semin Liver Dis. 2006;26:42-51.

47 Zen Y, Fujii T, Sato Y, Masuda S, Nakanuma Y. Pathological classification of hepatic inflammatory pseudotumor with respect to IgG4-related disease. Mod Pathol. 2007;20: 884-94.

48 Sempoux C, Fan C, Singh P, Obeidat K, Roayaie S, Schwartz M, et al. Cholangiolocellular carcinoma: an innocent-looking malignant liver tumor mimicking ductular reaction. Semin Liver Dis. 2011;31:104-10.

49 Bhathal PS, Hughes NR, Goodman ZD. The so-called bile duct adenoma is a peribiliary gland hamartoma. Am J Surg Pathol. 1996;20: 858-64.
Clinical Practice Guidelines for

Intrahepatic Cholangiocarcinoma
Liver Cancer 2022;11:290-314

DOI: $10.1159 / 000522403$ 
50 Karahan OI, Kahriman G, Soyuer I, Ok E. Hepatic von meyenburg complex simulating biliary cystadenocarcinoma. Clin Imaging. 2007;31:50-3.

51 Zen Y, Terahata S, Miyayama S, Mitsui T, Takehara A, Miura S, et al. Multicystic biliary hamartoma: a hitherto undescribed lesion. Hum Pathol. 2006;37:339-44.

52 Aljiffry M, Abdulelah A, Walsh M, Peltekian K, Alwayn I, Molinari M. Evidence-based approach to cholangiocarcinoma: a systematic review of the current literature. J Am Coll Surg. 2009;208:134-47.

53 DeOliveira ML, Cunningham SC, Cameron JL, Kamangar F, Winter JM, Lillemoe KD, et al. Cholangiocarcinoma: thirty-one-year experience with 564 patients at a single institution. Ann Surg. 2007;245:755-62.

54 Kubo S, Takemura S, Tanaka S, Nishioka T, Kinoshita M, Hamano G, et al. Screening and surveillance for occupational cholangiocarcinoma in workers exposed to organic solvents. Surg Today. 2016;46:705-12.

55 Pasanen P, Pikkarainen P, Alhava E, Partanen $\mathrm{K}$, Pentila I. Value of serum alkaline phosphatase, aminotransferases, gamma-glutamyl transferase, leucine aminopeptidase, and bilirubin in the distinction between benign and malignant diseases causing jaundice and cholestasis: results from a prospective study. Scand J Clin Lab Invest. 1993;53:35-9.

56 Bain VG, Abraham N, Jhangri GS, Alexander TW, Henning RC, Hoskinson ME, et al. Prospective study of biliary strictures to determine the predictors of malignancy. Can J Gastroenterol. 2000;14:397-402.

57 Al-Mofleh IA, Aljebreen AM, Al-Amri SM, Al-Rashed RS, Al-Faleh FZ, Al-Freihi HM, et al. Biochemical and radiological predictors of malignant biliary strictures. World J Gastroenterol. 2004;10:1504-7.

58 Saluja SS, Sharma R, Pal S, Sahni P, Chattopadhyay TK. Differentiation between benign and malignant hilar obstructions using laboratory and radiological investigations: a prospective study. HPB. 2007;9:373-82.

59 Garcea G, Ngu W, Neal CP, Dennison AR, Berry DP. Bilirubin levels predict malignancy in patients with obstructive jaundice. HPB. 2011;13:426-30.

60 Qiu Y, He J, Chen X, Huang P, Hu K, Yan H. The diagnostic value of five serum tumor markers for patients with cholangiocarcinoma. Clin Chim Acta. 2018;480:186-92.

61 Chung YE, Kim MJ, Park YN, et al. Varying appearances of cholangiocarcinoma: radiologic - pathologic correlation. Radiographics. 2009;29:683-700.

62 Terminology and diagnostic criteria committee, Japan Society of Ultrasonics in Medicine. Ultrasound diagnostic criteria for hepatic tumors. J Med Ultrason. 2014;41:113-23.

63 Chen LD, Xu HX, Xie XY, Xie XH, Xu ZF, Liu GJ, et al. Intrahepatic cholangiocarcinoma and hepatocellular carcinoma: differential diagnosis with contrast-enhanced ultrasound. Eur Radiol. 2010;20:743-53.
64 Furuse J, Nagase M, Ishii H, Yoshino M. Contrast enhancement patterns of hepatic tumours during the vascular phase using coded harmonic imaging and Levovist to differentiate hepatocellular carcinoma from other focal lesions. Br J Radiol. 2003;76:385-92.

65 Shin SK, Choi DJ, Kim JH, Kim YS, Kwon OS. Characteristics of contrast-enhanced ultrasound in distinguishing small $(\leq 3 \mathrm{~cm})$ hepatocellular carcinoma from intrahepatic cholangiocarcinoma. Medicine. 2018;97:e12781.

66 Choi SH, Lee SS, Kim SY, Park SH, Park SH, $\mathrm{Kim} \mathrm{KM}$, et al. Intrahepatic cholangiocarcinoma in patients with cirrhosis: differentiation from hepatocellular carcinoma by using gadoxetic acid-enhanced MR imaging and dynamic CT. Radiology. 2017;282:771-81.

67 Kim SJ, Lee JM, Han JK, Kim KH, Lee JY, Choi BI. Peripheral mass-forming cholangiocarcinoma in cirrhotic liver. AJR Am J Roentgenol. 2007;189:1428-34.

68 Zhang Y, Uchida M, Abe T, Nishimura $H$, Hayabuchi N, Nakashima Y. Intrahepatic peripheral cholangiocarcinoma: comparison of dynamic CT and dynamic MRI. J Comput Assist Tomogr. 1999;23:670-7.

69 Jeong HT, Kim MJ, Chung YE, Choi JY, Park YN, Kim KW. Gadoxetate disodium-enhanced MRI of mass-forming intrahepatic cholangiocarcinomas: imaging-histologic correlation. AJR Am J Roentgenol. 2013;201: W603-11.

70 Peporte AR, Sommer WH, Nikolaou K, Reiser MF, Zech CJ. Imaging features of intrahepatic cholangiocarcinoma in Gd-EOB-DTPA-enhanced MRI. Eur J Radiol. 2013;82: e101-6.

71 Kim R, Lee JM, Shin CI, Lee ES, Yoon JH, Joo $\mathrm{I}$, et al. Differentiation of intrahepatic massforming cholangiocarcinoma from hepatocellular carcinoma on gadoxetic acid-enhanced liver MR imaging. Eur Radiol. 2016; 26:1808-17

72 Huang B, Wu L, Lu XY, Xu F, Liu CF, Shen WF, et al. Small intrahepatic cholangiocarcinoma and hepatocellular carcinoma in cirrhotic livers may share similar enhancement patterns at multiphase dynamic MR imaging. Radiology. 2016;281:150-7.

73 Park HJ, Jang KM, Kang TW, Song KD, Kim $\mathrm{SH}$, Kim YK, et al. Identification of imaging predictors discriminating different primary liver tumours in patients with chronic liver disease on gadoxetic acid-enhanced MRI: a classification tree analysis. Eur Radiol. 2016; 26:3102-11.

74 Lewis S, Besa C, Wagner M, Jhaveri K, Kihira $\mathrm{S}$, Zhu H, et al. Prediction of the histopathologic findings of intrahepatic cholangiocarcinoma: qualitative and quantitative assessment of diffusion-weighted imaging. Eur Radiol. 2018;28:2047-57.
75 Wengert GJ, Baltzer PAT, Bickel H, Thurner P, Breitenseher J, Lazar M, et al. Differentiation of intrahepatic cholangiocellular carcinoma from hepatocellular carcinoma in the cirrhotic liver using contrastenhanced MR imaging. Acad Radiol. 2017;24:1491-500.

76 Choi SY, Kim YK, Min JH, Kang TW, Jeong WK, Ahn S, et al. Added value of ancillary imaging features for differentiating scirrhous hepatocellular carcinoma from intrahepatic cholangiocarcinoma on gadoxetic acid-enhanced MR imaging. Eur Radiol. 2018;28: 2549-60.

77 Hwang J, Kim YK, Min JH, Choi SY, Jeong WK, Hong SS, et al. Capsule, septum, and T2 hyperintense foci for differentiation between large hepatocellular carcinoma $(\geq 5 \mathrm{~cm})$ and intrahepatic cholangiocarcinoma on gadoxetic acid MRI. Eur Radiol. 2017;27:4581-90.

78 Corvera CU, Blumgart LH, Akhurst T, DeMatteo RP, D’Angelica M, Fong Y, et al. 18Ffluorodeoxyglucose positron emission tomography influences management decisions in patients with biliary cancer. J Am Coll Surg. 2008;206:57-65.

79 Kim YJ, Yun M, Lee WJ, Kim KS, Lee JD. Usefulness of 18F-FDG PET in intrahepatic cholangiocarcinoma. Eur J Nucl Med Mol Imaging. 2003;30:1467-72.

80 Anderson CD, Rice MH, Pinson CW, Chapman WC, Chari RS, Delbeke D. Fluorodeoxyglucose PET imaging in the evaluation of gallbladder carcinoma and cholangiocarcinoma. J Gastrointest Surg. 2004;8:90-7.

81 Moon CM, Bang S, Chung JB, Park SW, Song SY, Yun M, et al. Usefulness of 18F-fluorodeoxyglucose positron emission tomography in differential diagnosis and staging of cholangiocarcinomas. J Gastroenterol Hepatol. 2008;23:759-65.

82 Sammon J, Fischer S, Menezes R, HosseiniNik H, Lewis S, Taouli B, et al. MRI features of combined hepatocellular-cholangiocarcinoma versus mass forming intrahepatic cholangiocarcinoma. Cancer Imaging. 2018;18:8.

83 Asayama Y, Yoshimitsu K, Irie H, Tajima T, Nishie A, Hirakawa M, et al. Delayed-phase dynamic CT enhancement as a prognostic factor for mass-forming intrahepatic cholangiocarcinoma. Radiology. 2006;238:150-5.

84 Chen L, Zhang J, Zhang L, Bao J, Liu C, Xia Y, et al. Meta-analysis of gadoxetic acid disodium (Gd-EOB-DTPA)-enhanced magnetic resonance imaging for the detection of liver metastases. PLoS One. 2012;7:e48681.

85 Sugimoto K, Shiraishi J, Moriyasu F, Saito K. Improved detection of hepatic metastases with contrast-enhanced low mechanical-index pulse inversion ultrasonography during the liver-specific phase of sonazoid: observer performance study with JAFROC analysis Acad Radiol. 2009;16:798-809.

86 Mavros MN, Economopoulos KP, Alexiou VG, Pawlik TM. Treatment and prognosis for patients with intrahepatic cholangiocarcinoma: systematic review and meta-analysis. JAMA Surg. 2014;149:565-74. 
87 Ruys AT, van Beem BE, Engelbrecht MR, Bipat S, Stoker J, Van Gulik TM. Radiological staging in patients with hilar cholangiocarcinoma: a systematic review and meta-analysis. Br J Radiol. 2012;85:1255-62.

88 Park HS, Lee JM, Choi JY, Lee MW, Kim HJ, Han JK, et al. Preoperative evaluation of bile duct cancer: MRI combined with MR cholangiopancreatography versus MDCT with direct cholangiography. AJR Am J Roentgenol. 2008;190:396-405.

89 Mohamadnejad M, DeWitt JM, Sherman S, LeBlanc JK, Pitt HA, House MG, et al. Role of EUS for preoperative evaluation of cholangiocarcinoma: a large single-center experience. Gastrointest Endosc. 2011;73:71-8.

90 Tsukada K, Takada T, Miyazaki M, Miyakawa S, Nagino M, Kondo S, et al. Diagnosis of biliary tract and ampullary carcinomas. J Hepatobiliary Pancreat Surg. 2008;15:31-40.

91 Yao S, Taura K, Okuda Y, Kodama Y, Uza N, Gouda N, et al. Effect of mapping biopsy on surgical management of cholangiocarcinoma. J Surg Oncol. 2018;118:997-1005.

92 Ito K, Sakamoto Y, Isayama H, Nakai Y, Watadani T, Tanaka M, et al. The impact of MDCT and endoscopic transpapillary mapping biopsy to predict longitudinal spread of extrahepatic cholangiocarcinoma. J Gastrointest Surg. 2018;22:1528-37.

93 Ogawa T, Ito K, Koshita S, Kanno Y, Masu K, Kusunose $\mathrm{H}$, et al. Usefulness of cholangioscopic-guided mapping biopsy using SpyGlass DS for preoperative evaluation of extrahepatic cholangiocarcinoma: a pilot study. Endosc Int Open. 2018;6:E199-204.

94 Park TG, Yu YD, Park BJ, Cheon GJ, Oh SY, Kim DS, et al. Implication of lymph node metastasis detected on 18F-FDG PET区CT for surgical planning in patients with peripheral intrahepatic cholangiocarcinoma. Clin Nucl Med. 2014;39:1-7.

95 Holzapfel K, Gaa J, Schubert EC, Eiber M, Kleeff J, Rummeny EJ, et al. Value of diffusion-weighted MR imaging in the diagnosis of lymph node metastases in patients with cholangiocarcinoma. Abdom Radiol 2016;41:1937-41.

96 Songthamwat M, Chamadol N, Khuntikeo N, Thinkhamrop J, Koonmee S, Chaichaya N, et al. Evaluating a preoperative protocol that includes magnetic resonance imaging for lymph node metastasis in the cholangiocarcinoma screening and care program (CASCAP) in Thailand. World J Surg Oncol. 2017;15:176.

97 Jiang L, Tan H, Panje CM, Yu H, Xiu Y, Shi $\mathrm{H}$. Role of 18F-FDG PET/CT imaging in intrahepatic cholangiocarcinoma. Clin Nucl Med. 2016;41:1-7.

98 Ma KW, Cheung TT, She WH, Chok KSH, Chan ACY, Dai WC, et al. Diagnostic and prognostic role of 18-FDG PET/CT in the management of resectable biliary tract cancer. World J Surg. 2018;42:823-34.

99 Som PM. Detection of metastasis in cervical lymph nodes: CT and MR criteria and differential diagnosis. AJR Am J Roentgenol. 1992; 158:961-9.
100 Wu W, He X, Andayani D, Yang L, Ye J, Li $\mathrm{Y}$, et al. Pattern of distant extrahepatic metastases in primary liver cancer: a SEER based study. J Cancer. 2017;8:2312-8.

101 Weber SM, Ribero D, O’Reilly EM, Kokudo $\mathrm{N}$, Miyazaki M, Pawlik TM. Intrahepatic cholangiocarcinoma: expert consensus statement. HPB. 2015;17:669-80.

102 Petrowsky H, Wildbrett P, Husarik DB, Hany TF, Tam S, Jochum W, et al. Impact of integrated positron emission tomography and computed tomography on staging and management of gallbladder cancer and cholangiocarcinoma. J Hepatol. 2006;45: 43-50.

103 Kim JY, Kim MH, Lee TY, Hwang CY, Kim JS, Yun SC, et al. Clinical role of $18 \mathrm{~F}-\mathrm{FDG}$ PET-CT in suspected and potentially operable cholangiocarcinoma: a prospective study compared with conventional imaging. Am J Gastroenterol. 2008;103:1145-51.

104 Lee SW, Kim HJ, Park JH, Park DI, Cho YK, Sohn CI, et al. Clinical usefulness of 18FFDG PET-CT for patients with gall- bladder cancer and cholangiocarcinoma. J Gastroenterol. 2010;45:560-6.

105 Kim SA, Lee JM, Lee KB, Kim SH, Yoon SH, $\mathrm{Han} \mathrm{JK}$, et al. Intrahepatic mass-forming cholangiocarcinomas: enhancement patterns at multiphasic CT, with special emphasis on arterial enhancement patterncorrelation with clinicopathologic findings. Radiology. 2011;260:148-57.

106 Iwasa S, Morizane C, Okusaka T, Ueno H, Ikeda M, Kondo S, et al. Cisplatin and etoposide as first-line chemotherapy for poorly differentiated neuroendocrine carcinoma of the hepatobiliary tract and pancreas. Jpn J Clin Oncol. 2010;40:313-8.

107 Buscarini L, Fornari F, Bolondi L, Colombo $\mathrm{P}$, Livraghi T, Magnolfi F, et al. Ultrasoundguided fine-needle biopsy of focal liver lesions: techniques, diagnostic accuracy and complications. A retrospective study on 2,091 biopsies. J Hepatol. 1990;11:344-8.

108 Silva MA, Hegab B, Hyde C, Guo B, Buckels JA, Mirza DF. Needle track seeding following biopsy of liver lesions in the diagnosis of hepatocellular cancer: a systematic review and meta-analysis. Gut. 2008;57:1592-6.

109 Yamasaki S. Intrahepatic cholangiocarcinoma: macroscopic type and stage classification. J Hepatobiliary Pancreat Surg. 2003; 10:288-91.

110 Lim JH. Cholangiocarcinoma: morphologic classification according to growth pattern and imaging findings. AJR Am J Roentgenol. 2003;181:819-27.

111 Morimoto Y, Tanaka Y, Ito T, Nakahara M, Nakaba H, Nishida T, et al. Long-term survival and prognostic factors in the surgical treatment for intrahepatic cholangiocarcinoma. J Hepatobiliary Pancreat Surg. 2003; 10:432-40.
112 Nakanuma Y, Harada K, Ishikawa A, Zen Y, Sasaki M. Anatomic and molecular pathology of intrahepatic cholangiocarcinoma. J Hepatobiliary Pancreat Surg. 2003;10:26581.

113 Uchiyama K, Yamamoto M, Yamaue $\mathrm{H}$, Ariizumi S, Aoki T, Kokudo N, et al. Impact of nodal involvement on surgical outcomes of intrahepatic cholangiocarcinoma: a multicenter analysis by the study group for hepatic surgery of the Japanese society of hepato-biliary-pancreatic surgery. J Hepatobiliary Pancreat Sci. 2011;18:443-52.

114 Yamamoto M, Ariizumi S. Surgical outcomes of intrahepatic cholangiocarcinoma. Surg Today. 2011;41:896-902.

115 Imai K, Yamamoto M, Ariizumi S. Surgery for periductal infiltrating type intrahepatic cholangiocarcinoma without hilar invasion provides a better outcome than for massforming type intrahepatic cholangiocarcinoma without hilar invasion. Hepatogastroenterology. 2010;57:1333-6.

116 Okabayashi T, Yamamoto J, Kosuge T, Shimada K, Yamasaki S, Takayama T, et al. A new staging system for mass-forming intrahepatic cholangiocarcinoma: analysis of preoperative and postoperative variables. Cancer. 2001;92:2374-83.

117 Shimada K, Sano T, Sakamoto Y, Esaki M, Kosuge T, Ojima H. Surgical outcomes of the mass: forming plus periductal infiltrating types of intrahepatic cholangiocarcinoma: a comparative study with the typical mass-forming type of intrahepatic cholangiocarcinoma. World J Surg. 2007;31:201622.

118 Paik KY, Jung JC, Heo JS, Choi SH, Choi DW, Kim YI. What prognostic factors are important for resected intrahepatic cholangiocarcinoma? J Gastroenterol Hepatol. 2008;23:766-70.

119 Shimada K, Sano T, Nara S, Esaki M, Sakamoto $\mathrm{Y}$, Kosuge T, et al. Therapeutic value of lymph node dissection during hepatectomy in patients with intrahepatic cholangiocellular carcinoma with negative lymph node involvement. Surgery. 2009;145:4116.

120 Guglielmi A, Ruzzenente A, Campagnaro T, Pachera S, Valdegamberi A, Nicoli P, et al. Intrahepatic cholangiocarcinoma: prognostic factors after surgical resection. World Surg. 2009;33:1247-54

121 Ariizumi S, Kotera Y, Takahashi Y, Katagiri $S$, Chen IP, Ota T, et al. Mass-forming intrahepatic cholangiocarcinoma with marked enhancement on arterial-phase computed tomography reflects favorable surgical outcomes. J Surg Oncol. 2011;104:130-9.

122 Suzuki S, Sakaguchi T, Yokoi Y, Okomato $\mathrm{K}$, Kurachi K, Tsuchiya Y, et al. Clinicopathological prognostic factors and impact of surgical treatment of mass-forming intrahepatic cholangiocarcinoma. World J Surg. 2002;26:687-93.
Clinical Practice Guidelines for

Intrahepatic Cholangiocarcinoma
Liver Cancer 2022;11:290-314 
123 Nathan H, Aloia TA, Vauthey JN, Abdalla EK, Zhu AX, Schulick RD, et al. A proposed staging system for intrahepatic cholangiocarcinoma. Ann Surg Oncol. 2009;16:14-22.

124 Uenishi T, Kubo S, Yamazaki O, Yamada T, Sasaki Y, Nagano H, et al. Indications for surgical treatment of intrahepatic cholangiocarcinoma with lymph node metastases. J Hepatobiliary Pancreat Surg. 2008; 15: $417-22$.

125 Nakagawa T, Kamiyama T, Kurauchi N, Matsushita M, Nakanishi K, Kamachi H, et al. Number of lymph node metastases is a significant prognostic factor in intrahepatic cholangiocarcinoma. World J Surg. 2005; 29:728-33.

126 Sapisochin G, Facciuto M, Rubbia-Brandt $\mathrm{L}$, et al. Liver transplantation for "very early" intra- hepatic cholangiocarcinoma: international retrospective study supporting a prospective assessment. Hepatology. 2016;64: 1178-88.

127 Farges O, Fuks D, Boleslawski E, Le Treut YP, Castaing D, Laurent A, et al. Influence of surgical margins on outcome in patients with intrahepatic cholangiocarcinoma: a multicenter study by the AFC-IHCC-2009 study group. Ann Surg. 2011;254:824-30.

128 Spolverato G, Yakoob MY, Kim Y, Alexandrescu S, Marques HP, Lamelas J, et al. The impact of surgical margin status on longterm outcome after resection for intrahepatic cholangiocarcinoma. Ann Surg Oncol. 2015;22:4020-8.

129 Li MX, Bi XY, Li ZY, Huang Z, Han Y, Zhao JJ, et al. Impaction of surgical margin status on the survival outcome after surgical resection of intrahepatic cholangiocarcinoma: a systematic review and meta-analysis. J Surg Res. 2016;203:163-73.

130 Zhang XF, Bagante F, Chakedis J, Moris D, Beal EW, Weiss M, et al. Perioperative and long-term outcome for intrahepatic cholangiocarcinoma: impact of major versus minor hepatectomy. J Gastrointest Surg. 2017; 21:1841-50.

131 Kinoshita M, Kanazawa A, Takemura S, Tanaka S, Kodai S, Shinkawa H, et al. Indications for laparoscopic liver resection of mass-forming intrahepatic cholangiocarcinoma. Asian J Endosc Surg. 2020;13:46-58.

132 Liver Cancer Study Group of Japan. General rules for the clinical and pathological study of primary liver cancer. 2nd ed. Tokyo: Kanehara \& Co., Ltd.; 2003.

133 Sahara K, Tsilimigras DI, Merath K, Bagante F, Guglielmi A, Aldrighetti L, et al. Therapeutic index associated with lymphadenectomy among patients with intrahepatic cholangiocarcinoma: Which patients benefit the most from nodal evaluation? Ann Surg Oncol. 2019;26:2959-68.

134 Kang CM, Suh KS, Yi NJ, Hong TH, Park SJ, Ahn KS, et al. Should lynph nodes be retrieved in patients with intrahepatic cholangiocarcinoma? A collaborative Korea-Japan study. Cancers. 2021;13:445.
135 Zhang XF, Lv Y, Weiss M, Popescu I, Marques HP, Aldrighetti L, et al. Should utilization of lymphadenectomy vary according to morphologic subtype of intrahepatic cholangiocarcinoma? Ann Surg Oncol. 2019;26:2242-50.

136 Kim DH, Choi DW, Choi SH, Heo JS, Kow $\mathrm{AW}$. Is there a role for systematic hepatic pedicle lymphadenectomy in intrahepatic cholangiocarcinoma? A review of 17 years of experience in a tertiary institution. Surgery. 2015;157:666-75.

137 Li DY, Zhang HB, Yang N, Quan Y, Yang GS. Routine lymph node dissection may be not suitable for all intra- hepatic cholangiocarcinoma patients: results of a monocentric series. World J Gastroenterol. 2013;19: 9084-91.

138 Morine Y, Shimada M. The value of systematic lymph node dissection for intrahepatic cholangiocarcinoma from the viewpoint of liver lymphatics. J Gastroenterol. 2015;50: 913-27.

139 Hu J, Chen FY, Zhou KQ, Zhou C, Cao Y, Sun HC, et al. Intrahepatic cholangiocarcinoma patients without indications of lymph node metastasis not benefit from lymph node dissection. Oncotarget. 2017;8: 113817-27.

140 Bagante F, Spolverato G, Weiss M, Alexandrescu S, Marques HP, Aldrighetti L, et al. Surgical management of intrahepatic cholangiocarcinoma in patients with cirrhosis: impact of lymphadenectomy on peri-operative outcomes. World J Surg. 2018;42: 2551-60.

141 Kim SH, Han DH, Choi GH, Choi JS, Kim KS. Oncologic impact of lymph node dissection for intrahepatic cholangiocarcinoma: a propensity score-matched study. J Gastrointest Surg. 2019;23:538-44.

142 Vitale A, Moustafa M, Spolverato G, Gani F, Cillo U, Pawlik TM. Defining the possible therapeutic benefit of lymphadenectomy among patients undergoing hepatic resection for intrahepatic cholangiocarcinoma. J Surg Oncol. 2016;113:685-91.

143 Zhang XF, Chakedis J, Bagante F, Chen Q, Beal EW, Lv Y, et al. Trends in use of lymphadenectomy in surgery with curative intent for intrahepatic cholangiocarcinoma. $\mathrm{Br} \mathrm{J}$ Surg. 2018;105:857-66.

144 Tan JCC, Coburn NG, Baxter NN, Kiss A, Law CH. Surgical management of intrahepatic cholangiocarcinoma-a population-based study. Ann Surg Oncol. 2008;15:600-8.

145 Amini N, Ejaz A, Spolverato G, et al. Temporal trends in liver-directed therapy of patients with intrahepatic cholangiocarcinoma in the United States: a population-based analysis. J Surg Oncol. 2014;110:163-70.

146 Kolarich AR, Shah JL, George TJ Jr, Hughes SJ, Shaw CM, Geller BS, et al. Non-surgical management of patients with intrahepatic cholangiocarcinoma in the United States, 2004-2015: an NCDB analysis. J Gastrointest Oncol. 2018;9:536-45.
147 Zhang SJ, Hu P, Wang N, Shen Q, Sun AX Kuang M, et al. Thermal ablation versus repeated hepatic resection for recurrent intrahepatic cholangiocarcinoma. Ann Surg Oncol. 2013;20:3596-602.

148 Valle J, Wasan H, Palmer DH, Cunningham D, Anthoney A, Maraveyas A, et al. Cisplatin plus gemcitabine versus gemcitabine for biliary tract cancer. N Engl J Med. 2010;362: 1273-81.

149 Okusaka T, Nakachi K, Fukutomi A, Mizuno N, Ohkawa S, Funakoshi A, et al. Gemcitabine alone or in combination with cisplatin in patients with biliary tract cancer: a comparative multicentre study in Japan. Br J Cancer. 2010;103:469-74.

150 Valle JW, Furuse J, Jitlal M, Beare S, Mizuno $\mathrm{N}$, Wasan $\mathrm{H}$, et al. Cisplatin and gemcitabine for advanced biliary tract cancer: a meta-analysis of two randomized trials. Ann Oncol. 2014;25:391-8.

151 Morizane C, Okusaka T, Mizusawa J, Katayama $\mathrm{H}$, Ueno M, Ikeda M, et al. Combination gemcitabine plus S-1 versus gemcitabine plus cisplatin for advanced/recurrent biliary tract cancer: the FUGA-BT (JCOG1113) randomized phase III clinical trial. Ann Oncol. 2019;30:1950-8.

152 Shroff RT, Javle MM, Xiao L, Kaseb AO, Varadhachary GR, Wolff RA, et al. Gemcitabine, cisplatin, and nab-paclitaxel for the treatment of advanced biliary tract cancers: a phase 2 clinical trial. JAMA Oncol. 2019;5:824-30

153 Sharma A, Dwary AD, Mohanti BK, Deo SV, Pal S, Sreenivas V, et al. Best supportive care compared with chemotherapy for unresectable gall bladder cancer: a randomized controlled study. J Clin Oncol. 2010;28: 4581-6.

154 Falkson G, MacIntyre JM, Moertel CG Eastern cooperative oncology group experience with chemotherapy for inoperable gallbladder and bile duct cancer. Cancer. 1984; 54:965-9.

155 Takada T, Kato H, Matsushiro T, Nimura Y, Nagakawa T, Nakayama T. Comparison of 5-fluorouracil, doxorubicin and mitomycin $\mathrm{C}$ with 5-fluorouracil alone in the treatment of pancreatic-biliary carcinomas. Oncology. 1994;51:396-400.

156 Glimelius B, Hoffman K, Sjödén PO, Jacobsson G, Sellström H, Enander LK, et al. Chemotherapy improves survival and quality of life in advanced pancreatic and biliary cancer. Ann Oncol. 1996;7:593-600.

157 Raderer M, Hejna MH, Valencak JB, Kornek GV, Weinländer GS, Bareck E, et al. Two consecutive phase II studies of 5-fluorouracil/leucovorin/mitomycin $\mathrm{C}$ and of gemcitabine in patients with advanced biliary cancer. Oncology. 1999;56:177-80. 
158 Kornek GV, Schuell B, Laengle F, Gruenberger T, Penz M, Karall K, et al. Mitomycin $\mathrm{C}$ in combination with capecitabine or biweekly high-dose gemcitabine in patients with advanced biliary tract cancer: a randomized phase II trial. Ann Oncol. 2004;15: 478-83.

159 Ducreux M, Van Cutsem E, Van Laethem JL, Gress TM, Jeziorski K, Rougier P, et al. A randomised phase II trial of weekly highdose 5-fluorouracil with and without folinic acid and cisplatin in patients with advanced biliary tract carcinoma: results of the 40955 EORTC trial. Eur J Cancer. 2005;41:398403.

160 Rao S, Cunningham D, Hawkins RE, Hill ME, Smith D, Daniel F, et al. Phase III study of 5FU, etoposide and leucovorin (FELV) compared to epirubicin, cisplatin and 5FU (ECF) in previously untreated patients with advanced biliary cancer. Br J Cancer. 2005; 92:1650-4.

161 Lee J, Park SH, Chang HM, Kim JS, Choi HJ, Lee MA, et al. Gemcitabine and oxaliplatin with or without erlotinib in advanced biliary-tract cancer: a multicentre, open-label, randomized, phase 3 study. Lancet Oncol. 2012;13:181-8.

162 Sakai D, Kanai M, Kobayashi S, Hatano E, Nagano H, Iona T, et al. Randomized phase III study of gemcitabine, cisplatin plus S-1 (GCS) versus gemcitabine, cisplatin (GC) for advanced biliary tract cancer $(\mathrm{KH}-$ BO1401-MITSUBA). Ann Oncol. 2018; 29(Suppl 8):viii205-70.

163 Kobayashi S, Ueno M, Ohkawa S, Andou T, Kameda R, Yamamoto N, et al. A retrospective study of S-1 monotherapy as secondline treatment for patients with advanced biliary tract cancer. Jpn J Clin Oncol. 2012; 42:800-6.

164 Katayose Y, Ohtsuka H, Kitamura Y, Masuda K, Nakagawa K, Yamamoto K, et al. An analysis of a second-line S-1 monotherapy for gemcitabine-refractory biliary tract cancer. Hepatogastroenterology. 2012;59:6915.

165 Sasaki T, Isayama H, Nakai Y, Mizuno S, Yamamoto $\mathrm{K}$, Yagioka $\mathrm{H}$, et al. Multicenter phase II study of S-1 monotherapy as second-line chemotherapy for advanced biliary tract cancer refractory to gemcitabine. Invest New Drugs. 2012;30:708-13.

166 Le DT, Durham JN, Smith KN, Wang H, Bartlett BR, Aulakh LK, et al. Mismatch repair deficiency predicts response of solid tumors to PD-1 blockade. Science. 2017;357: 409-13.

167 Doebele RC, Drilon A, Paz-Ares L, Siena S, Shaw AT, Farago AF, et al. Entrectinib in patients with advanced or metastatic NTRK fusion-positive solid tumours: integrated analysis of three phase 1-2 trials. Lancet Oncol. 2020;21:271-82.
168 Ross JS, Wang K, Gay L, Al-Rohil R, Rand JV, Jones DM, et al. New routes to targeted therapy of intrahepatic cholangiocarcinomas revealed by next-generation sequencing. Oncologist. 2014;19:235-42.

169 Abou-Alfa GK, Sahai V, Hollebecque A, Vaccaro G, Melisi D, Al-Rajabi R, et al. Pemigatinib for previously treated, locally advanced or metastatic cholangiocarcinoma: a multicentre, open-label, phase 2 study. Lancet Oncol. 2020;21:671-85.

170 Arai Y, Totoki Y, Hosoda F, Shirota T, Hama N, Nakamura H, et al. Fibroblast growth factor receptor 2 tyrosine kinase fusions define a unique molecular subtype of cholangiocarcinoma. Hepatology. 2014;59: 1427-34.

171 Maruki Y, Morizane C, Arai Y, Ikeda M, Ueno M, Ioka T, et al. Molecular detection and clinicopathological characteristics of advanced/recurrent biliary tract carcinomas harboring the FGFR2 rearrangements: a prospective observational study (PRELUDE study). J Gastroenterol. 2021;56:250-60.

172 Tsujie M, Iwai T, Kubo S, Ura T, Hatano E, Sakai D, et al. Fibroblast growth factor receptor 2 (FGFR2) fusions in Japanese patients with intrahepatic cholangiocarcinoma. Jpn J Clin Oncol. 2021;51:911-7.

173 Abou-Alfa GK, Macarulla Mercade T, Javle M, Macarulla T, Javle RM, Kelley RK, et al. ClarIDHy: a global, phase 3, randomized, double-blind study of ivosidenib versus placebo in patients with advanced cholangiocarcinoma with an isocitrate dehydrogenase 1 mutation. Ann Oncol. 2019;30(5):v851.

174 Luo X, Yuan L, Wang Y, Ge R, Sun Y, Wei G. Survival outcomes and prognostic factors of surgical therapy for all potentially resectable intrahepatic cholangiocarcinoma: a large single-center cohort study. J Gastrointest Surg. 2014;18:562-72.

175 Kamo N, Mori A, Nitta T, Hatano E, Mitsuyoshi $\mathrm{H}$, Ikeda $\mathrm{K}$, et al. Two cases of curatively resected intrahepatic cholangiocellular carcinomas through effective response to neoadjuvant chemotherapy. Gan To Kagakuryoho. 2011;38:305-8.

176 Kato A, Shimizu H, Ohtsuka M, Allard MA, Pereira B, Serji B, et al. Neoadjuvant downsizing chemotherapy following conversion surgery for initially unresectable locally advanced intrahepatic cholangiocarcinoma. Kan·Tan·Sui. 2016;72:85-90.

177 Kato A, Shimizu H, Ohtsuka M, Yoshidome $\mathrm{H}$, Yoshitomi H, Furukawa K, et al. Surgical resection after downsizing chemotherapy for initially unresectable locally advanced biliary tract cancer: a retrospective singlecenter study. Ann Surg Oncol. 2013;20:31824.

178 Le Roy B, Gelli M, Pittau G, Allard MA, Pereira B, Serji B, et al. Neoadjuvant chemotherapy for initially unresectable intrahepatic cholangiocarcinoma. Br J Surg. 2018; 105:839-47.
179 Reames BN, Bagante F, Ejaz A, Spolverato G, Ruzzenente A, Weiss M, et al. Impact of adjuvant chemotherapy on survival in patients with intrahepatic cholangiocarcinoma: a multi-institutional analysis. HPB. 2017;19:901-9.

180 Luvira V, Eurboonyanun CH, Bhudhisawasdi V, Pugkhem A, Pairojkul CH, Luvira $\mathrm{V}$, et al. Patterns of recurrence after resection of mass-forming type intrahepatic cholangiocarcinomas. Asian Pac J Cancer Prev. 2016;17:4735-9.

181 Horgan AM, Amir E, Walter T, Knox JJ. Adjuvant therapy in the treatment of biliary tract cancer: a systematic review and metaanalysis. J Clin Oncol. 2012;30:1934-40.

182 Ma KW, Cheung TT, Leung B, She BWH, Chok KSH, Chan ACY, et al. Adjuvant chemotherapy improves oncological outcomes of resectable intrahepatic cholangiocarcinoma: a meta-analysis. Medicine. 2019;98: e14013.

183 Kobayashi S, Nagano H, Sakai D, Eguchi H, Hatano E, Kanai M, et al. Phase I study of adjuvant gemcitabine or S-1 in patients with biliary tract cancers undergoing major hepatectomy: KHBO1003 study. Cancer Chemother Pharmacol. 2014;74:699-709.

184 Kainuma O, Miura F, Furukawa D, Yamamoto H, Cho A, Sano K, et al. Feasibility and efficacy of gemcitabine plus cisplatin combination therapy after curative resection for biliary tract cancer. J Hepatobiliary Pancreat Sci. 2015;22:789-94.

185 Fujiwara Y, Kobayashi S, Nagano H, Kanai M, Hatano E, Toyoda M, et al. Pharmacokinetic study of adjuvant gemcitabine therapy for biliary tract cancer following major hepatectomy (KHBO1101). PLoS One. 2015;10: e0143072.

186 Siebenhüner AR, Seifert $H$, Bachmann $H$, Seifert B, Winder T, Feilchenfeldt J, et al. Adjuvant treatment of resectable biliary tract cancer with cisplatin plus gemcitabine: a prospective single center phase II study. BMC Cancer. 2018;18:72.

187 Woo SM, Yoon KA, Hong EK, Park WS, Han SS, Park SJ, et al. DCK expression, a potential predictive biomarker in the adjuvant gemcitabine chemotherapy for biliary tract cancer after surgical resection: results from a phase II study. Oncotarget. 2017;8: 81394-404.

188 Nakachi K, Konishi M, Ikeda M, Shimada K, Okusaka T, Saiura A, et al. Feasibility study of postoperative adjuvant chemotherapy with S-1 in patients with biliary tract cancer. Int J Clin Oncol. 2018;23:894-9.

189 Takada T, Amano H, Yasuda H, Nimura Y, Matsushiro T, Kato $\mathrm{H}$, et al. Is postoperative adjuvant chemotherapy useful for gallbladder carcinoma? A phase III multicenter prospective randomized controlled trial in patients with resected pancreaticobiliary carcinoma. Cancer. 2002;95:1685-95.
Clinical Practice Guidelines for

Intrahepatic Cholangiocarcinoma
Liver Cancer 2022;11:290-314 
190 Neoptolemos JP, Moore MJ, Cox TF, Valle JW, Palmer DH, McDonald AC, et al. Effect of adjuvant chemotherapy with fluorouracil plus folinic acid or gemcitabine versus observation on survival in patients with resected periampullary adenocarcinoma the ESPAC-3 periampullary cancer randomized trial. JAMA. 2012;308:147-56.

191 Ebata T, Hirano S, Konishi M, Uesaka K, Tsuchiya Y, Ohtsuka M, et al. Randomized clinical trial of adjuvant gemcitabine chemo- therapy versus observation in resected bile duct cancer. Br J Surg. 2018;105:192202.

192 Primrose JN, Fox RP, Palmer DH, Malik HZ, Prasad R, Mirza D, et al. BILCAP study group. Capecitabine compared with observation in resected biliary tract cancer (BILCAP): a randomised, controlled, multicentre, phase 3 study. Lancet Oncol. 2019;20: 663-73.

193 Edeline J, Benabdelghani M, Bertaut A, Watelet J, Hammel P, Joly JP, et al. Gemcitabine and oxaliplatin chemotherapy or surveillance in resected biliary tract cancer (PRODIGE 12-ACCORD 18-UNICANCER GI): a randomized phase III study. J Clin Oncol. 2019;37:658-67.

194 Kobayashi S, Nagano H, Tomokuni A, Gotoh K, Sakai D, Hatano E, et al. A prospective, randomized phase II study of adjuvant gemcitabine versus S-1 after major hepatectomy for biliary tract cancer (KHBO 1208): Kansai Hepato-Biliary Oncology Group. Ann Surg. 2019;270:230-7.

195 Shroff RT, Kennedy EB, Bachini M, BekaiiSaab T, Crane C, Edeline J, et al. Adjuvant therapy for resected biliary tract cancer: ASCO clinical practice guideline. J Clin Oncol. 2019;37:1015-27.

196 Ikeda K, Nakachi M, Konishi S, Mizusawa J, Eba J, Okusaka T, et al. A randomized phase III trial comparing adjuvant chemotherapy with S-1 vs. surgery alone in patients with resectable biliary tract cancer: JCOG 1202 (ASCOT). J Clin Oncol. 2017;35:TPS4144.

197 Stein A, Arnold D, Bridgewater J. Adjuvant chemotherapy with gemcitabine and cisplatin compared to observation after curative intent resection of cholangiocarcinoma and muscle invasive gallbladder carcinoma (ACTICCA-1 trial)-a randomized, multidisciplinary, multinational phase III trial. BMC Cancer. 2015; 15:564.

198 Tse RV, Hawkins M, Lockwood G, Kim JJ, Cummings B, Knox J, et al. Phase I study of individualized stereotactic body radiotherapy for hepatocellular carcinoma and intrahepatic cholangiocarcinoma. J Clin Oncol. 2008;26:657-64.

199 Kopek N, Holt MI, Hansen AT, Hoyer M. Stereotactic body radiotherapy for unresectable cholangiocarcinoma. Radiother Oncol. 2010;94:47-52.

200 Tao R, Krishnan S, Bhosale PR, Javle MM, Aloia TA, Shroff RT, et al. Ablative radiotherapy doses lead to a substantial prolongation of survival in patients with inoperable intrahepatic cholangiocarcinoma: a retrospective dose response analysis. J Clin Oncol. 2016;34:219-26.

201 Shen ZT, Zhou H, Li AM, Li B, Shen JS, Zhu XX. Clinical outcomes and prognostic factors of stereotactic body radiation therapy for intrahepatic cholangiocarcinoma. Oncotarget. 2017;8:93541-50.

202 Weiner AA, Olsen J, Ma D, Dyk P, DeWees $\mathrm{T}$, Myerson RJ, et al. Stereotactic body radiotherapy for primary hepatic malignancies-report of a phasee I/II institutional study. Radiother Oncol. 2016;121:79-85.

203 Jung DH, Kim MS, Cho CK, Yoo HJ, Jang WI, Seo YS, et al. Outcomes of stereotactic body radiotherapy for unresectable primary or recurrent cholangiocarcinoma. Radiat Oncol J. 2014;32:163-9.
204 Ohkawa A, Mizumoto M, Ishikawa H, Abei M, Fukuda K, Hashimoto T, et al. Proton beam therapy for unresectable intrahepatic cholangiocarcinoma. J Gastroenterol Hepatol. 2015;30:957-63.

205 Hong TS, Wo JY, Yeap BY, Ben-Josef E, McDonnell EI, Blaszkowsky LS, et al. Multi-institutional phase II study of high-dose hypofractionated proton beam therapy in patients with localized, unresectable hepatocellular carcinoma and intrahepatic cholangiocarcinoma. J Clin Oncol. 2016;34:460-8.

206 Abe T, Shibuya K, Koyama Y, Okamoto M, Kiyohara H, Katoh H, et al. Initial results of hypofractionated carbon ion radiotherapy for cholangiocarcinoma. Anticancer Res. 2016;36:2955-60.

207 Makita C, Nakamura T, Takada A, Takayama K, Suzuki M, Ishikawa Y, et al. Clinical outcomes and toxicity of proton beam therapy for advanced cholangiocarcinoma. Radiat Oncol. 2014;9:26.

208 Kasuya G, Terashima K, Shibuya K, Toyama $\mathrm{S}$, Ebner DK, Tsuji H, et al. Carbon-ion radiotherapy for cholangiocarcinoma: a multi-institutional study by and the Japan carbon-ion radiation oncology study group (J-CROS). Oncotarget. 2019;10:4369-79.

209 Sobin LH, Gospodarowicz MK, Wittekind C, editors. TNM classification of malignant tumors. 7th ed. Oxford: Wiley Blackwell; 2009.

210 Classification of Biliary Tract Cancer (sixth). Japanese society of biliary surgery. Tokyo: Kanehara \& Co., Ltd; 2013.

211 Ojima H. Current state and future perspective of histological classification of intrahepatic cholangiocarcinoma. Kan tan sui. 2016;72:39-49.

212 Nakamura H, Arai Y, Totoki Y, Shirota T, Elzawahry A, Kato M, et al. Genomic spectra of biliary tract cancer. Nat Genet. 2015; 47:1003-10. 